\title{
A stochastic network equilibrium model for electric power markets with uncertain demand*
}

\author{
Massimo Pappalardo ${ }^{\dagger} \quad$ Mauro Passacantando ${ }^{\ddagger} \quad$ Fabio Raciti ${ }^{\S}$
}

\begin{abstract}
We use the theory of stochastic variational inequalities to develop a network equilibrium model of the whole supply chain of electricity markets: power generation, supply, transmission, and consumption. In particular, we take into account the case where the market demand functions are not exactly known but are affected by some kind of uncertainty. A discretization and truncation procedure is used to numerically solve the stochastic variational inequality model. Monotonicity properties of the operator are investigated and the affine case is analyzed in detail. Finally, numerical experiments show the impact of different probability densities of the random variables on the approximated solutions and the scalability of the proposed numerical method for real-world sized problems.
\end{abstract}

Keywords: Stochastic variational inequality, Electricity market, Random demand.

\section{Introduction}

Electricity market models have been developed at a very high pace in the last fifteen years, also due to the fact that in many countries the electricity power industry has undergone a transformation from a government-regulated to a competitive regime. In such a regime there are several classes of decision makers: power generators, power suppliers and consumers. Providers that ensure the power transmission can also be included in the modeling but usually they are not considered as decision makers. The complexity of the interaction among these three different groups in the decision process has thus required advanced mathematical tools. Two main approaches have been developed in the literature to address the deregulated electric power markets: deterministic equilibrium models (where the power demand is known in a deterministic manner) and stochastic programming models (where the power demand is affected by some kind of uncertainty).

The first approach is based on complementarity and variational inequality models that describe in a unified way the simultaneous optimization problems which arise at the different decision tiers. The reader interested in complementarity models for energy markets can refer to the excellent tutorial [26] which also provides numerous related references. A detailed

\footnotetext{
${ }^{*}$ This paper has been published in Optimization, 2019, DOI: 10.1080/02331934.2019.1671383. This work was supported by University of Pisa (grant number PRA-2017-05)

${ }^{\dagger}$ Department of Computer Science, University of Pisa, Largo B. Pontecorvo 3, 56127 Pisa, Italy, email: massimo.pappalardo@unipi.it

${ }^{\ddagger}$ Department of Computer Science, University of Pisa, Largo B. Pontecorvo 3, 56127 Pisa, Italy, email: mauro.passacantando@unpi.it

${ }^{\S}$ Department of Mathematics and Computer Science, University of Catania, Viale A. Doria 6, 95125 Catania, Italy, email: fraciti@dmi.unict.it
} 
variational inequality model of the restructured electricity system can be found in [7], while for models which utilize both Nash equilibrium concepts and variational inequalities we refer to [23]. In [22], the authors provide a network variational inequality model which simultaneously describe both the optimization problems of the power generators and the power suppliers, and the equilibrium conditions at the consumer markets.

The second approach is based on the use of stochastic programming techniques in order to take into account the uncertainty of the power demand. In [27], nonlinear stochastic programming methods are used to provide a decision support tool for optimizing the expansion planning of a semi-liberalized electricity market. In [6], the authors consider a game model of electricity markets with one Gaussian random demand, where each player solves an optimization problem under chance constraints. The book [5] contains a detailed survey on electricity market models with uncertain data, with stochastic programming techniques, but does not cover the variational inequality approach. In [1], stochastic programming techniques are used to incorporate uncertain wind generation into the European market electricity. In the very recent paper [11], the authors investigate a stochastic oligopoly model which takes into account long-term infrastructure investment decisions. They used the so called open-loop approach, meaning that investments and market operation (daily) decisions are assumed to be made simultaneously. From the technical point of view, their approach is a generalization of the classical two-stage stochastic programming to the case of multiple decision makers.

The first approach has the advantage of considering the equilibrium conditions of the whole chain of generation, supply and consumption of electric power, but has the inconvenience of assuming that the demand for electricity can be predicted with certainty. On the other hand, the second approach takes into account the inherent uncertainty of the power demand, but has the drawback of using stochastic optimization techniques instead of general equilibrium conditions that are more suitable to describe the whole system.

The aim of our paper is to merge the best features of both approaches, that is we introduce a stochastic equilibrium model for the whole chain of generation, supply and consumption of electric power, assuming that the power demand is uncertain. In particular, we model the stochastic equilibrium as the solution of a system of three stochastic variational inequalities; such a system is proved to be equivalent (under mild assumptions) to a single stochastic variational inequality; we show that the stochastic variational inequality model can be numerically solved by a well-known approximation/discretization procedure; we investigate in detail the monotonicity properties of the map of the stochastic variational inequality, which are useful to prove the convergence of the approximation procedure; the affine variational inequality case, which is the most common in applications, is deeply analyzed. Finally, several numerical experiments show the viability of our approach: we show the convergence of mean values and standard deviations of the approximated solutions, the impact of different probability densities of the random variables on the approximated solutions and how the approximation procedure is able to solve real-world size problems.

The modeling of market demands as random variables is well established in Economics and in Finance, as is shown by numerous papers addressing this topic in the last decades (see e.g. $[8,12,24])$. The causes of demand uncertainty may result from specific aspects of the business and its customers or from external factors. Moreover, any innovation in technology or service will face a great deal of demand uncertainty, simply because there is no previous statistical analysis from which to draw conclusions about demand. Demand can also be reallocated, in an uncertain way, by the entry of new competitors into the industry. Natural or human-caused disasters and times of political unrest are examples of external factors that 
contribute to both demand and supply uncertainty. In the specific case of electricity markets, random fluctuations of the temperature in summer or winter cause an increase in the electricity power needed to run heating and air conditioning or cooling systems. By using historical time series analysis, temperature probability distributions can be derived and possibly used to model the random demand of electric power. This is particularly relevant for those countries where heating is mostly based on electricity (for example France). Moreover, malfunctions or disruptions of technical nature occur randomly and can be modeled if detailed knowledge of the robustness and reliability of the various parts of the generation-distribution chain is available.

Let us recall that while the theory of stochastic programming is a well established field of optimization [25], stochastic (or random) formulations of variational inequalities are much more recent although this topic has undergone a great development in the last decade. In particular, we exploit in this paper the rigorous approach to random variational inequalities which has been put forward in $[13,14,15,16]$ and applied to several equilibrium problems $[4$, $9,17]$. Stochastic variational inequalities have also been considered as particular applications of the methodology of variational convergence for bifunctions studied in [18], but in a finite dimensional setting.

This paper is organized as follows. In Section 2, we describe in detail the model, define an equilibrium for the whole chain of generation, supply and consumption of electric power, and derive the random variational inequality that describes the equilibrium conditions of the markets involved. In Section 3, we summarize the theoretical framework of random variational inequalities and outline an approximation procedure for their solution. Section 4 is devoted to investigate the monotonicity properties of the operator which appears in the variational inequality, which are useful to prove the convergence of our approximation procedure. Section 5 analyzes in detail the affine case, while Section 6 is devoted to numerical experiments. Conclusions are finally drawn in Section 7.

\section{The model}

In this section, we describe the whole chain of generation, supply and consumption of electric power with random demand and derive a stochastic variational inequality model that describes the equilibrium conditions of the markets involved. A detailed economic explanation of all the processes involved in the electricity market, assuming that the power demand is known in a deterministic manner, can be found in [22]. We also remark that the model proposed in [22] includes some constraints in the operator, while in our approach we prefer to explicitly consider the constraints, both to reduce the number of variables involved and to make the study of monotonicity properties of the operator clearer and more connected with the economic model.

The markets' structure is modeled by a network with three tiers of nodes representing the different decision makers: power generators, power suppliers and consumers (see Figure 1). The network flows do not describe the physical electric power flows, but the corresponding economic transactions. Moreover, we do not take into account the physical power transmission network, which makes our model particularly suitable for long-term planning problems. However, the random demand functions to be introduced in the sequel (or possible random upper bounds on the flows) could incorporate (although in an aggregate, not explicit manner) 


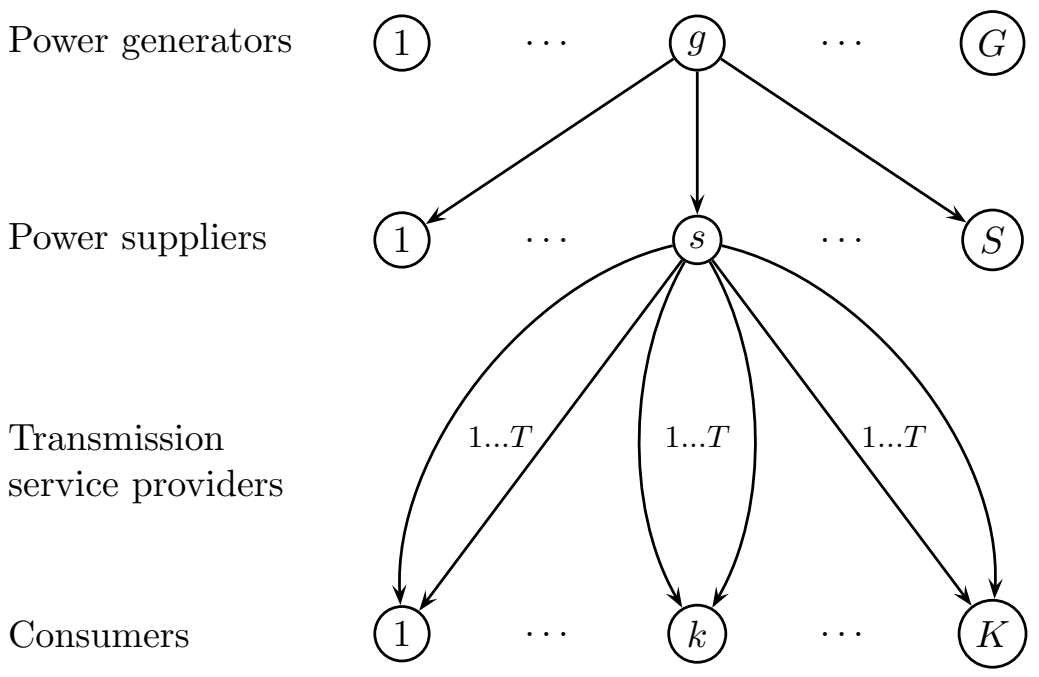

Figure 1: The electric power supply chain.

the effect of possible issues connected to malfunctions in the physical network.

Power distribution is ensured by transmission service providers which do not take part in the decision process. There are $G$ power generators in the first tier of nodes and $S$ power suppliers in the second tier. The role of suppliers is to intermediate the transactions between generators and consumers, which are described by the third tier. Suppliers pay the transmission services to ensure the physical distribution of electric power. There are $T$ transmission services, $K$ demand markets and the decision makers of each tier are in competition.

Let $g$ denote a power generator, $Q_{g s}^{1}$ the power transacted from generator $g$ to supplier $s$ and $\rho_{g s}^{1}$ the unit price that $g$ charges $s$. The flows and prices between power generators and suppliers are grouped in two vectors $Q^{1}, \rho^{1} \in \mathbb{R}_{+}^{G S}$, respectively. Let $Q_{s k t}^{2}$ denote the amount of electricity transacted between supplier $s$ and consumer $k$ via the link corresponding to the transmission provider $t$ and $\rho_{s k t}^{2}$ the unit price associated with the transaction from $s$ to $k$ via $t$. The transaction flows and prices are grouped in two vectors $Q^{2}, \rho^{2} \in \mathbb{R}_{+}^{S K T}$, respectively. In the following, we describe the optimization problems and the related equilibrium conditions for each tier.

\subsection{Equilibrium conditions for the consumers}

We start from the bottom level of the network, i.e. the consumers, since we assume that their demand functions are uncertain and this will affect the equilibrium values of variables (flows and prices) of the other tiers. Let $\rho_{k}^{3}$ denote the unit price of electric power associated with the demand market $k$ and assume that its demand $d_{k}$ can depend in general on the prices at all markets $\rho^{3}=\left(\rho_{1}^{3}, \ldots, \rho_{K}^{3}\right)$, so as to model competition. As explained in the introduction, seasonal fluctuations in the temperature, possible malfunctions in the network, or changes in the buying strategy of consumers, suggest that the demand can fluctuate randomly.

Hence, we assume that $d_{k}$ is a random variable, that is $d_{k}: \Omega \times \mathbb{R}^{K} \rightarrow \mathbb{R}$, where $\Omega$ is the sample space with a probability measure $P$. Let $\hat{c}_{s k t}=\hat{c}_{s k t}\left(Q^{2}\right)$ denote the unit transaction cost between the demand market $k$ and the supplier $s$ via the transmission provider $t$. Since 
the demand functions are random variables, an equilibrium between the demand markets and the suppliers can be defined as a random vector $\left(Q^{2 *}(\omega), \rho^{3 *}(\omega)\right)$ such that for each $s, k, t$ and for $P$-almost every $\omega \in \Omega$ the following relations hold:

$$
d_{k}\left(\omega, \rho^{3 *}(\omega)\right) \begin{cases}=\sum_{s=1}^{S} \sum_{t=1}^{T} Q_{s k t}^{2 *}(\omega) & \text { if } \rho_{k}^{3 *}(\omega)>0, \\ \leq \sum_{s=1}^{S} \sum_{t=1}^{T} Q_{s k t}^{2 *}(\omega) & \text { if } \rho_{k}^{3 *}(\omega)=0,\end{cases}
$$

and

$$
\rho_{s k t}^{2 *}(\omega)+\hat{c}_{s k t}\left(Q^{2 *}(\omega)\right) \begin{cases}=\rho_{k}^{3 *}(\omega) & \text { if } Q_{s k t}^{2 *}(\omega)>0, \\ \geq \rho_{k}^{3 *}(\omega) & \text { if } Q_{s k t}^{2 *}(\omega)=0 .\end{cases}
$$

Equilibrium conditions of this kind (in a deterministic setting) are quite standard in the modeling of spatially distributed markets (see e.g. [21, Chapter 3]). We remark that prices are not random variables in our model, but equilibrium prices at the consumer markets $\rho_{k}^{3 *}(\omega)$ are random because the demand is random. Moreover, notice that also the equilibrium prices $\rho_{g s}^{1 *}(\omega)$ and $\rho_{s k t}^{2 *}(\omega)$ are considered as random variables because of the uncertainty of the demand functions. Anyhow, the values of $\rho^{1 *}(\omega)$ and $\rho^{2 *}(\omega)$ can be derived from the solution of the variational inequality (4) introduced at the end of this section through the relations (5) and (6), hence in the above equations they have the role of random parameters.

The above equilibrium conditions can be expressed in a compact form as the following stochastic variational inequality: for $P$-almost every $\omega \in \Omega$, find $\left(Q^{2 *}(\omega), \rho^{3 *}(\omega)\right) \in \mathbb{R}_{+}^{S K T+K}$ such that

$$
\begin{aligned}
& \sum_{s=1}^{S} \sum_{k=1}^{K} \sum_{t=1}^{T}\left[\rho_{s k t}^{2 *}(\omega)+\hat{c}_{s k t}\left(Q^{2 *}(\omega)\right)-\rho_{k}^{3 *}(\omega)\right] \cdot\left[Q_{s k t}^{2}-Q_{s k t}^{2 *}(\omega)\right] \\
& +\sum_{k=1}^{K}\left[\sum_{s=1}^{S} \sum_{t=1}^{T} Q_{s k t}^{2 *}(\omega)-d_{k}\left(\omega, \rho^{3 *}(\omega)\right)\right] \cdot\left[\rho_{k}^{3}-\rho_{k}^{3 *}(\omega)\right] \geq 0, \quad \forall\left(Q^{2}, \rho^{3}\right) \in \mathbb{R}_{+}^{S K T+K} .
\end{aligned}
$$

\subsection{Equilibrium conditions for the power suppliers}

The suppliers acquire electricity from the power generators and sell it to the consumers via the transmission providers. There are several kinds of costs which are faced by a supplier $s$ and are globally referred to as operating costs and denoted by $c_{s}$. Assume that the operating costs depend on both $Q^{1}$ and $Q^{2}$, that is $c_{s}=c_{s}\left(Q^{1}, Q^{2}\right)$. Moreover, we denote the part of transaction cost with the power generator $g$ paid by the supplier $s$ with $\hat{c}_{g s}=\hat{c}_{g s}\left(Q^{1}\right)$. We also consider the transaction $\operatorname{cost} c_{s k t}=c_{s k t}\left(Q^{2}\right)$ paid by the supplier $s$ to the transmission service $t$ in order to dispatch the power to the consumer $k$. The above cost functions are assumed to be convex and continuously differentiable.

Since each supplier $s$ is a profit-maximizer, it aims to solve, for every $\omega \in \Omega$, the following 
optimization problem:

$$
\begin{aligned}
\max & \sum_{k=1}^{K} \sum_{t=1}^{T} \rho_{s k t}^{2 *}(\omega) Q_{s k t}^{2}-c_{s}\left(Q^{1}, Q^{2}\right)-\sum_{g=1}^{G} \rho_{g s}^{1 *}(\omega) Q_{g s}^{1}-\sum_{g=1}^{G} \hat{c}_{g s}\left(Q^{1}\right)-\sum_{k=1}^{K} \sum_{t=1}^{T} c_{s k t}\left(Q^{2}\right) \\
\text { subject to: } & \sum_{k=1}^{K} \sum_{t=1}^{T} Q_{s k t}^{2} \leq \sum_{g=1}^{G} Q_{g s}^{1} \\
& Q_{g s}^{1} \geq 0 \quad \forall g=1, \ldots, G, \\
& Q_{s k t}^{2} \geq 0 \quad \forall k=1, \ldots, K, t=1, \ldots, T .
\end{aligned}
$$

The objective function represents the difference between the revenue due to the sell to the consumers and the total cost, while the first constraint imposes that the total quantity of electricity sold to the consumers is compatible with that bought from the generators.

The equilibrium conditions for all the suppliers can be equivalently described by the following stochastic variational inequality: for $P$-almost every $\omega \in \Omega$, find $\left(Q^{1 *}(\omega), Q^{2 *}(\omega)\right) \in$ $C_{s}$ such that:

$$
\begin{aligned}
& \sum_{g=1}^{G} \sum_{s=1}^{S}\left[\frac{\partial c_{s}\left(Q^{1 *}(\omega), Q^{2 *}(\omega)\right)}{\partial Q_{g s}^{1}}+\frac{\partial \hat{c}_{g s}\left(Q^{1 *}(\omega)\right)}{\partial Q_{g s}^{1}}+\rho_{g s}^{1 *}(\omega)\right] \cdot\left[Q_{g s}^{1}-Q_{g s}^{1 *}(\omega)\right] \\
& +\sum_{s=1}^{S} \sum_{k=1}^{K} \sum_{t=1}^{T}\left[\frac{\partial c_{s}\left(Q^{1 *}(\omega), Q^{2 *}(\omega)\right)}{\partial Q_{s k t}^{2}}+\frac{\partial c_{s k t}\left(Q^{2 *}(\omega)\right)}{\partial Q_{s k t}^{2}}-\rho_{s k t}^{2 *}(\omega)\right] \cdot\left[Q_{s k t}^{2}-Q_{s k t}^{2 *}(\omega)\right] \geq 0
\end{aligned}
$$

holds for any $\left(Q^{1}, Q^{2}\right) \in C_{s}$, where

$$
C_{s}=\left\{\left(Q^{1}, Q^{2}\right) \in \mathbb{R}_{+}^{G S+S K T}: \sum_{k=1}^{K} \sum_{t=1}^{T} Q_{s k t}^{2} \leq \sum_{g=1}^{G} Q_{g s}^{1}, \quad \forall s=1, \ldots, S\right\} .
$$

\subsection{Equilibrium conditions for the power generators}

Let $q_{g}$ denote the power output (expressed in watts) of generator $g$ and the power outputs of all generators are grouped in a vector $q \in \mathbb{R}_{+}^{G}$. The cost of power generation is given by the function $f_{g}=f_{g}(q)$ which is assumed to be convex and continuously differentiable for any $g=1, \ldots, G$. The generators also pay part of the cost associated to power transmission to the suppliers. These costs are described by the convex and continuously differentiable cost functions $c_{g s}=c_{g s}\left(Q^{1}\right)$ for any $g$ and $s$. The flow conservation law $q_{g}=\sum_{s=1}^{S} Q_{g s}^{1}$ allows writing $f_{g}=f_{g}\left(Q^{1}\right)$ for any $g$. Since each generator $g$ is a profit-maximizer, it wants to solve, for every $\omega \in \Omega$, the following optimization problem:

$$
\begin{aligned}
\max & \sum_{s=1}^{S} \rho_{g s}^{1 *}(\omega) Q_{g s}^{1}-f_{g}\left(Q^{1}\right)-\sum_{s=1}^{S} c_{g s}\left(Q^{1}\right) \\
\text { subject to: } & Q_{g s}^{1} \geq 0 \quad \forall s=1, \ldots, S .
\end{aligned}
$$

The simultaneous solution of the above optimization problems for all the generators is equivalent to the following stochastic variational inequality: for $P$-almost every $\omega \in \Omega$, find $Q^{1 *}(\omega) \in \mathbb{R}_{+}^{G S}$ such that

$$
\sum_{g=1}^{G} \sum_{s=1}^{S}\left[\frac{\partial f_{g}\left(Q^{1 *}(\omega)\right)}{\partial Q_{g s}^{1}}+\frac{\partial c_{g s}\left(Q^{1 *}(\omega)\right)}{\partial Q_{g s}^{1}}-\rho_{g s}^{1 *}(\omega)\right] \cdot\left[Q_{g s}^{1}-Q_{g s}^{1 *}(\omega)\right] \geq 0, \quad \forall Q^{1} \in \mathbb{R}_{+}^{G S}
$$




\subsection{Equilibrium conditions for the whole chain}

The variational inequalities (1), (2) and (3) describe the noncooperative behavior of the decision makers at each tier and the economic subjects at different tiers share some groups of variables. The following equilibrium definition for the whole supply chain is then natural.

Definition 2.1. A random vector $\left(Q^{1 *}(\omega), Q^{2 *}(\omega), \rho^{3 *}(\omega)\right)$ is a supply chain equilibrium if (1), (2) and (3) hold simultaneously for some prices $\rho^{1 *}(\omega), \rho^{2 *}(\omega)$.

The supply chain equilibria are related to the solutions of a single stochastic variational inequality, as the following result shows.

Theorem 2.1. If $\left(Q^{1 *}(\omega), Q^{2 *}(\omega), \rho_{3}^{*}(\omega)\right)$ is a supply chain equilibrium, then it solves the following stochastic variational inequality: for P-almost every $\omega \in \Omega,\left(Q^{1 *}(\omega), Q^{2 *}(\omega), \rho_{3}^{*}(\omega)\right) \in$ $C:=C_{s} \times \mathbb{R}_{+}^{K}$ and

$$
\begin{aligned}
& \sum_{g=1}^{G} \sum_{s=1}^{S}\left[\frac{\partial f_{g}\left(Q^{1 *}(\omega)\right)}{\partial Q_{g s}^{1}}+\frac{\partial c_{g s}\left(Q^{1 *}(\omega)\right)}{\partial Q_{g s}^{1}}+\frac{\partial c_{s}\left(Q^{1 *}(\omega), Q^{2 *}(\omega)\right)}{\partial Q_{g s}^{1}}+\frac{\partial \hat{c}_{g s}\left(Q^{1 *}(\omega)\right)}{\partial Q_{g s}^{1}}\right] \cdot\left[Q_{g s}^{1}-Q_{g s}^{1 *}(\omega)\right] \\
& +\sum_{s=1}^{S} \sum_{k=1}^{K} \sum_{t=1}^{T}\left[\frac{\partial c_{s}\left(Q^{1 *}(\omega), Q^{2 *}(\omega)\right)}{\partial Q_{s k t}^{2}}+\frac{\partial c_{s k t}\left(Q^{2 *}(\omega)\right)}{\partial Q_{s k t}^{2}}+\hat{c}_{s k t}\left(Q^{2 *}(\omega)\right)-\rho_{k}^{3 *}(\omega)\right] \cdot\left[Q_{s k t}^{2}-Q_{s k t}^{2 *}(\omega)\right] \\
& +\sum_{k=1}^{K}\left[\sum_{s=1}^{S} \sum_{t=1}^{T} Q_{s k t}^{2 *}(\omega)-d_{k}\left(\omega, \rho^{3 *}(\omega)\right)\right] \cdot\left[\rho_{k}^{3}-\rho_{k}^{3 *}(\omega)\right] \geq 0, \quad \forall\left(Q^{1}, Q^{2}, \rho^{3}\right) \in C .
\end{aligned}
$$

Conversely, if $\left(Q^{1 *}(\omega), Q^{2 *}(\omega), \rho^{3 *}(\omega)\right)$ solves the stochastic variational inequality (4) and the prices $\rho^{1 *}(\omega)$ and $\rho^{2 *}(\omega)$ are defined as follows:

$$
\begin{array}{rlrl}
\rho_{g s}^{1 *}(\omega) & :=\frac{\partial f_{g}\left(Q^{1 *}(\omega)\right)}{\partial Q_{g s}^{1}}+\frac{\partial c_{g s}\left(Q^{1 *}(\omega)\right)}{\partial Q_{g s}^{1}}, & \forall g & =1, \ldots, G, s=1, \ldots, S, \\
\rho_{s k t}^{2 *}(\omega) & :=\rho_{k}^{3 *}(\omega)-\hat{c}_{s k t}\left(Q^{2 *}(\omega)\right), & \forall s=1, \ldots, S, k=1, \ldots, K, t=1, \ldots, T,
\end{array}
$$

then $\left(Q^{1 *}(\omega), Q^{2 *}(\omega), \rho^{3 *}(\omega)\right)$ is a supply chain equilibrium.

Proof. The first part follows from the hypotheses in a straightforward fashion since (4) is the sum of variational inequalities (1), (2) and (3).

For the second part, if in (4) we set $Q^{1}=Q^{1 *}(\omega)$ and $Q^{2}=Q^{2 *}(\omega)$, then

$$
\sum_{k=1}^{K}\left[\sum_{s=1}^{S} \sum_{t=1}^{T} Q_{s k t}^{2 *}(\omega)-d_{k}\left(\omega, \rho^{3 *}(\omega)\right)\right] \cdot\left[\rho_{k}^{3}-\rho_{k}^{3 *}(\omega)\right] \geq 0,
$$

holds for any $\rho^{3} \geq 0$. Therefore, the validity of (1) follows from (6). Moreover, if in (4) we set $\rho^{3}=\rho^{3 *}(\omega)$, then

$$
\begin{aligned}
0 \leq & \sum_{g=1}^{G} \sum_{s=1}^{S}\left[\frac{\partial f_{g}\left(Q^{1 *}(\omega)\right)}{\partial Q_{g s}^{1}}+\frac{\partial c_{g s}\left(Q^{1 *}(\omega)\right)}{\partial Q_{g s}^{1}}+\frac{\partial c_{s}\left(Q^{1 *}(\omega), Q^{2 *}(\omega)\right)}{\partial Q_{g s}^{1}}+\frac{\partial \hat{c}_{g s}\left(Q^{1 *}(\omega)\right)}{\partial Q_{g s}^{1}}\right] \cdot\left[Q_{g s}^{1}-Q_{g s}^{1 *}(\omega)\right] \\
& +\sum_{s=1}^{S} \sum_{k=1}^{K} \sum_{t=1}^{T}\left[\frac{\partial c_{s}\left(Q^{1 *}(\omega), Q^{2 *}(\omega)\right)}{\partial Q_{s k t}^{2}}+\frac{\partial c_{s k t}\left(Q^{2 *}(\omega)\right)}{\partial Q_{s k t}^{2}}+\hat{c}_{s k t}\left(Q^{2 *}(\omega)\right)-\rho_{k}^{3 *}(\omega)\right] \cdot\left[Q_{s k t}^{2}-Q_{s k t}^{2 *}(\omega)\right] \\
= & \sum_{g=1}^{G} \sum_{s=1}^{S}\left[\frac{\partial c_{s}\left(Q^{1 *}(\omega), Q^{2 *}(\omega)\right)}{\partial Q_{g s}^{1}}+\frac{\partial \hat{c}_{g s}\left(Q^{1 *}(\omega)\right)}{\partial Q_{g s}^{1}}+\rho_{g s}^{1 *}(\omega)\right] \cdot\left[Q_{g s}^{1}-Q_{g s}^{1 *}(\omega)\right] \\
& +\sum_{s=1}^{S} \sum_{k=1}^{K} \sum_{t=1}^{T}\left[\frac{\partial c_{s}\left(Q^{1 *}(\omega), Q^{2 *}(\omega)\right)}{\partial Q_{s k t}^{2}}+\frac{\partial c_{s k t}\left(Q^{2 *}(\omega)\right)}{\partial Q_{s k t}^{2}}-\rho_{s k t}^{2 *}(\omega)\right] \cdot\left[Q_{s k t}^{2}-Q_{s k t}^{2 *}(\omega)\right]
\end{aligned}
$$


holds for any $\left(Q^{1}, Q^{2}\right) \in C_{s}$, that is (2) holds. Finally, (3) directly follows from (5).

We remark that the economic meaning of condition (5) is that, at equilibrium, the price that a power generator charges a power supplier must be equal to the sum of the marginal production cost plus the marginal transaction cost.

In the following, it will be useful to rewrite the variational inequality (4) in a compact form. We denote by $\langle a, b\rangle$ the Euclidean scalar product between two vectors $a, b$ and by $\|a\|$ the Euclidean norm of $a$. We define the vector of variables $x=\left(Q^{1}, Q^{2}, \rho^{3}\right)$ and the operator $F: \Omega \times \mathbb{R}^{G S+S K T+K} \rightarrow \mathbb{R}^{G S+S K T+K}$ as follows:

$$
F(\omega, x):=\left(F^{1}\left(Q^{1}, Q^{2}\right), F^{2}\left(Q^{1}, Q^{2}, \rho^{3}\right), F^{3}\left(\omega, Q^{2}, \rho^{3}\right)\right),
$$

where

$$
\begin{aligned}
F_{g s}^{1}\left(Q^{1}, Q^{2}\right) & =\frac{\partial f_{g}\left(Q^{1}\right)}{\partial Q_{g s}^{1}}+\frac{\partial c_{g s}\left(Q^{1}\right)}{\partial Q_{g s}^{1}}+\frac{\partial c_{s}\left(Q^{1}, Q^{2}\right)}{\partial Q_{g s}^{1}}+\frac{\partial \hat{c}_{g s}\left(Q^{1}\right)}{\partial Q_{g s}^{1}}, \\
F_{s k t}^{2}\left(Q^{1}, Q^{2}, \rho^{3}\right) & =\frac{\partial c_{s}\left(Q^{1}, Q^{2}\right)}{\partial Q_{s k t}^{2}}+\frac{\partial c_{s k t}\left(Q^{2}\right)}{\partial Q_{s k t}^{2}}+\hat{c}_{s k t}\left(Q^{2}\right)-\rho_{k}^{3}, \\
F_{k}^{3}\left(\omega, Q^{2}, \rho^{3}\right) & =\sum_{s=1}^{S} \sum_{t=1}^{T} Q_{s k t}^{2}-d_{k}\left(\omega, \rho^{3}\right) .
\end{aligned}
$$

Then, the compact form of (4) is the following: for $P$-almost every $\omega \in \Omega$, find a random vector $x^{*}(\omega) \in C$ such that

$$
\left\langle F\left(\omega, x^{*}(\omega)\right), x-x^{*}(\omega)\right\rangle \geq 0, \quad \forall x \in C .
$$

Following $[13,15]$, instead of (8) we consider its integral version: find $x^{*} \in L^{p}$, with $p \geq 2$, such that $x^{*}(\omega) \in C$ for $P$-almost every $\omega \in \Omega$ and

$$
\int_{\Omega}\left\langle F\left(\omega, x^{*}(\omega)\right), x-x^{*}(\omega)\right\rangle d P(\omega) \geq 0
$$

holds for any $x \in L^{p}$ such that $x(\omega) \in C$ for $P$-almost every $\omega \in \Omega$. The reason to consider (9) is that since its solution $x^{*}=x^{*}(\omega)$ belongs to some $L^{p}$ space, (where $p \geq 2$ depends on $F(\omega, \cdot))$ it has finite first and second order moments. In particular, we are interested in computing the approximated mean value and variance of the solution with respect to the probability measure $P$. Notice that if problems (8) and (9) are uniquely solvable, then they are equivalent provided that the solution of (8) is an $L^{p}$ function (see Remark 3.1).

For the reader's convenience, we now recall the following general result (see, e.g., [20]) that ensures the solvability of an infinite dimensional variational inequality like (9).

Theorem 2.2. Let $E$ be a reflexive Banach space and let $E^{*}$ denote its topological dual space. We denote the duality pairing between $E$ and $E^{*}$ by $\langle\cdot, \cdot\rangle_{E, E^{*}}$. Let $K$ be a nonempty, closed, and convex subset of $E$ and $A: K \rightarrow E^{*}$ be monotone and continuous on finite dimensional subspaces of $K$. Consider the variational inequality problem of finding $u \in K$ such that

$$
\langle A u, v-u\rangle_{E, E^{*}} \geq 0, \quad \forall v \in K .
$$

Then, a necessary and sufficient condition for the above problem to be solvable is the existence of $\delta>0$ such that at least a solution of the variational inequality:

$$
\text { find } u_{\delta} \in K_{\delta} \text { such that }\left\langle A u_{\delta}, v-u_{\delta}\right\rangle_{E, E^{*}} \geq 0, \quad \forall v \in K_{\delta}
$$

satisfies $\left\|u_{\delta}\right\|<\delta$, where $K_{\delta}=\{v \in K:\|v\| \leq \delta\}$. 


\section{Methodology}

In this section, we briefly recall some basic notions about random variational inequalities of the kind (9) (see $[13,15]$ for more details). For the sake of simplicity, in what follows we set $N:=G S+S K T+K$ so that $x \in \mathbb{R}^{N}$ and $F: \Omega \times \mathbb{R}^{N} \rightarrow \mathbb{R}^{N}$. In our model we assume that the random and the deterministic variables appearing in $F$ are separated, i.e.,

$$
F(\omega, x)=Z(\omega) G(x)+H(x)-b-R(\omega) c,
$$

where $(\Omega, \mathcal{A}, P)$ is a probability space, $R$ and $Z$ are given real random variables defined on $\Omega$, the maps $G, H: \mathbb{R}^{N} \rightarrow \mathbb{R}^{N}$ are given and $b, c \in \mathbb{R}^{N}$ are two given vectors.

Consider the following stochastic variational inequality: for $P$-almost every $\omega \in \Omega$, find $\hat{x}:=\hat{x}(\omega) \in C$ such that

$$
\langle Z(\omega) G(\hat{x})+H(\hat{x}), x-\hat{x}\rangle \geq\langle R(\omega) c+b, x-\hat{x}\rangle, \quad \forall x \in C,
$$

where $C$ is a closed convex subset of $\mathbb{R}^{N}$. We assume that $Z, G$ and $H$ are such that $F$ is a Carathéodory function, that is, for each fixed $x \in \mathbb{R}^{N}$ the function $F(\cdot, x)$ is measurable with respect to $\mathcal{A}$, whereas for $P$-almost every $\omega \in \Omega$ the function $F(\omega, \cdot)$ is continuous.

Definition 3.1. Given any $\omega \in \Omega$, the map $F(\omega, \cdot)$ is monotone if and only if

$$
\langle F(\omega, x)-F(\omega, \tilde{x}), x-\tilde{x}\rangle \geq 0, \quad \forall x, \tilde{x} \in \mathbb{R}^{N} ;
$$

$F(\omega, \cdot)$ is strongly monotone uniformly with respect to $\omega \in \Omega$ if and only if there exists $\alpha>0$ such that

$$
\langle F(\omega, x)-F(\omega, \tilde{x}), x-\tilde{x}\rangle \geq \alpha\|x-\tilde{x}\|^{2}, \quad \forall \omega \in \Omega, \forall x, \tilde{x} \in \mathbb{R}^{N} .
$$

The strong monotonicity of $F(\omega, \cdot)$ is sufficient to ensure the existence of a unique solution to (11). Other sufficient conditions can be given to guarantee the existence of solutions, but the strong monotonicity also plays a crucial role in the convergence theorem at the end of this section and it is verified in several applications as shown in Section 4.

Since we are only interested in solutions with finite first and second order moments, we consider an integral variational inequality instead of the variational inequality (11). For a fixed $p \geq 2$, we consider the reflexive Banach space $L^{p}\left(\Omega, P, \mathbb{R}^{N}\right)$ of random vectors $V$ from $\Omega$ to $\mathbb{R}^{N}$ such that the expectation ( $p$-moment) is finite, that is

$$
E^{P}\left(\|V\|^{p}\right)=\int_{\Omega}\|V(\omega)\|^{p} d P(\omega)<\infty .
$$

For subsequent developments, we need the following growth condition

$$
\|F(\omega, x)\| \leq \alpha(\omega)+\beta(\omega)\|x\|^{p-1}, \quad \forall x \in \mathbb{R}^{N},
$$

where $\alpha \in L^{q}(\Omega, P)$ with $p^{-1}+q^{-1}=1$ and $\beta \in L^{\infty}(\Omega, P)$. Due to the above growth condition, the Nemytskii operator $\hat{F}$ associated to $F$, acts from $L^{p}\left(\Omega, P, \mathbb{R}^{N}\right)$ to $L^{q}\left(\Omega, P, \mathbb{R}^{N}\right)$ and is defined by

$$
\hat{F}(V)(\omega):=F(\omega, V(\omega)), \quad \omega \in \Omega .
$$


We introduce the following closed and convex set:

$$
C^{P}:=\left\{V \in L^{p}\left(\Omega, P, \mathbb{R}^{N}\right): V(\omega) \in C \text { for } P \text {-almost every } \omega \in \Omega\right\} .
$$

We assume that $R \in L^{q}(\Omega, P)$ and, without any loss of generality, it is nonnegative (otherwise we can use the standard decomposition in the positive part and the negative part). Moreover, we assume that $Z \in L^{\infty}(\Omega, P)$ and its support, i.e. the set of possible outcomes, is the interval $[\underline{z}, \bar{z}) \subset(0, \infty)$. Equipped with these notations, we consider the following $L^{p}$ formulation of (11): find $\hat{U} \in C^{P}$ such that

$$
\begin{aligned}
\int_{\Omega}\langle Z(\omega) & G(\hat{U}(\omega))+H(\hat{U}(\omega)), V(\omega)-\hat{U}(\omega)\rangle d P(\omega) \\
& \geq \int_{\Omega}\langle b+R(\omega) c, V(\omega)-\hat{U}(\omega)\rangle d P(\omega), \quad \forall V \in C^{P} .
\end{aligned}
$$

The integrals above are well posed due to the growth condition (12).

Remark 3.1. Problems (8) and (9) (and hence in particular problems (11) and (13)) are equivalent if their solution is unique, in the sense that from the integral formulation we obtain a pointwise solution that is only defined $P$-a.s. on the sample space $\Omega$ and that coincides there with the pointwise (i.e. parametric) solution of (8). Conversely, if the solution of (8) belongs to $L^{p}$, it also solves (9).

Furthermore, if $F(\omega, \cdot)$ is strictly monotone, so is $\hat{F}$, hence both (11) and (13) have a unique solution. If $F(\omega, \cdot)$ is strongly monotone, uniformly with respect to $\omega \in \Omega$, then $\hat{F}$ is strongly monotone and, again, both (11) and (13) have a unique solution. In the latter case, we can also prove norm convergence of our approximation procedure (see Theorem 3.1). It is also worth noticing that the uniform strong monotonicity holds in the affine case under suitable assumptions (see Section 5).

To get rid of the abstract sample space $\Omega$, we consider the joint distribution $\mathbb{P}$ of the random vector $(R, Z)$ and work with the special probability space $\left(\mathbb{R}^{2}, \mathcal{B}\left(\mathbb{R}^{2}\right), \mathbb{P}\right)$. For simplicity, we assume that $R$ and $Z$ are independent random variables. We set

$$
r=R(\omega), z=Z(\omega), y=(r, z) .
$$

The variational inequality (11) reads: for $\mathbb{P}$-almost every $y \in \mathbb{R}^{2}$, find $\hat{x}(y) \in C$ such that

$$
\langle z G(\hat{x}(y))+H(\hat{x}(y)), x-\hat{x}(y)\rangle \geq\langle r c+b, x-\hat{x}(y)\rangle, \quad \forall x \in C .
$$

In order to obtain the integral formulation of $(14)$, consider the space $L^{p}\left(\mathbb{R}^{2}, \mathbb{P}, \mathbb{R}^{N}\right)$ and introduce the closed and convex set

$$
C_{\mathbb{P}}:=\left\{v \in L^{p}\left(\mathbb{R}^{2}, \mathbb{P}, \mathbb{R}^{N}\right): \quad v(y) \in C \text { for } \mathbb{P} \text {-almost every } y \in \mathbb{R}^{2}\right\} .
$$

We also assume that the probability measures $P_{R}$ and $P_{Z}$ of $R$ and $Z$ have the probability densities $\varphi_{R}$ and $\varphi_{Z}$, respectively. Therefore, we have

$$
d P_{R}(r)=\varphi_{R}(r) d r, \quad d P_{Z}(z)=\varphi_{Z}(z) d z .
$$

Notice that $v \in L^{p}\left(\mathbb{R}^{2}, \mathbb{P}, \mathbb{R}^{N}\right)$ means that the function $(r, z) \mapsto \varphi_{R}(r) \varphi_{Z}(z)$ belongs to the Lebesgue space $L^{p}\left(\mathbb{R}^{2}, \mathbb{R}^{N}\right)$ with respect to the Lebesgue measure. 
Therefore, we can define the probabilistic integral variational inequality: find $\hat{u}:=\hat{u}(y) \in$ $C_{\mathbb{P}}$ such that

$$
\begin{aligned}
& \int_{0}^{\infty} \int_{\underline{z}}^{\bar{z}}\langle z G(\hat{u})+H(\hat{u}), v-\hat{u}\rangle \varphi_{R}(r) \varphi_{Z}(z) d y \\
& \quad \geq \int_{0}^{\infty} \int_{\underline{z}}^{\bar{z}}\langle b+r c, v-\hat{u}\rangle \varphi_{R}(r) \varphi_{Z}(z) d y, \quad \forall v \in C_{\mathbb{P}} .
\end{aligned}
$$

For the reader's convenience, we now provide some details on the numerical approximation of the solution $\hat{u}$ and show how to compute a sequence of step functions $\left\{\hat{u}_{n}\right\}$ which converges strongly in $L^{p}$ to $\hat{u}$. First, we need a discretization of the space $X:=L^{p}\left(\mathbb{R}^{2}, \mathbb{P}, \mathbb{R}^{N}\right)$. We introduce a sequence $\left\{\pi_{n}\right\}$ of partitions of the support

$$
\Upsilon:=[0, \infty) \cdot[\underline{z}, \bar{z})
$$

of the probability measure $\mathbb{P}$ induced by the random variables $R$ and $Z$. For this, we set $\pi_{n}=\left(\pi_{n}^{R}, \pi_{n}^{Z}\right)$, where

$$
\begin{aligned}
\pi_{n}^{R} & :=\left(r_{n}^{0}, \ldots, r_{n}^{N_{n}^{R}}\right), \\
\pi_{n}^{Z} & :=\left(z_{n}^{0}, \ldots, z_{n}^{N_{n}^{Z}}\right), \\
0 & =r_{n}^{0}<r_{n}^{1}<\ldots r_{n}^{N_{n}^{R}}=n, \\
\underline{z} & =z_{n}^{0}<z_{n}^{1}<\ldots z_{n}^{N_{n}^{Z}}=\bar{z}, \\
\left|\pi_{n}^{R}\right| & :=\max \left\{r_{n}^{i}-r_{n}^{i-1}: i=1, \ldots, N_{n}^{R}\right\} \rightarrow 0 \quad \text { for } n \rightarrow+\infty, \\
\left|\pi_{n}^{Z}\right| & :=\max \left\{z_{n}^{j}-z_{n}^{j-1}: j=1, \ldots, N_{n}^{Z}\right\} \rightarrow 0 \quad \text { for } n \rightarrow+\infty .
\end{aligned}
$$

These partitions give rise to the exhausting sequence $\left\{\Upsilon_{n}\right\}$ of subsets of $\Upsilon$, where each $\Upsilon_{n}$ is given by the finite disjoint union of the intervals:

$$
I_{i j}^{n}:=\left[r_{n}^{i-1}, r_{n}^{i}\right) \times\left[z_{n}^{j-1}, z_{n}^{j}\right)
$$

For each $n \in \mathbb{N}$, we consider the space of the $\mathbb{R}^{N}$-valued step functions on $\Upsilon_{n}$, extended by 0 outside of $\Upsilon_{n}$ :

$$
X_{n}:=\left\{v_{n}: \mathbb{R}^{2} \rightarrow \mathbb{R}^{N}: v_{n}(r, z)=\sum_{i=1}^{N_{n}^{R}} \sum_{j=1}^{N_{n}^{Z}} v_{i j}^{n} 1_{I_{i j}^{n}}(r, z), \quad \text { with } v_{i j}^{n} \in \mathbb{R}^{N}\right\}
$$

where $1_{I}$ denotes the $\{0,1\}$-valued characteristic function of the subset $I$. To approximate an arbitrary function $w \in L^{p}\left(\mathbb{R}^{2}, \mathbb{P}, \mathbb{R}\right)$, we employ the mean value truncation operator $\mu_{0}^{n}$ associated to the partition $\pi_{n}$ given by

$$
\mu_{0}^{n} w:=\sum_{i=1}^{N_{n}^{R}} \sum_{j=1}^{N_{n}^{Z}}\left(\mu_{i j}^{n} w\right) 1_{I_{i j}^{n}}
$$

where

$$
\mu_{i j}^{n} w:= \begin{cases}\frac{1}{\mathbb{P}\left(I_{i j}^{n}\right)} \int_{I_{i j}^{n}} w(y) d \mathbb{P}(y) & \text { if } \quad \mathbb{P}\left(I_{i j}^{n}\right)>0 \\ 0 & \text { otherwise. }\end{cases}
$$


Analogously, for a $L^{p}$ vector function $v=\left(v_{1}, \ldots, v_{N}\right)$, we define

$$
\mu_{0}^{n} v:=\left(\mu_{0}^{n} v_{1}, \ldots, \mu_{0}^{n} v_{N}\right),
$$

for which one can prove that $\mu_{0}^{n} v$ converges to $v$ in $L^{p}\left(\mathbb{R}^{2}, \mathbb{P}, \mathbb{R}^{N}\right)$. Since our objective is to approximate the random variables $R$ and $Z$, we introduce

$$
\rho_{n}=\sum_{i=1}^{N_{n}^{R}} r_{n}^{i-1} 1_{I_{i j}^{n}}, \quad \sigma_{n}=\sum_{j=1}^{N_{n}^{Z}} z_{n}^{j-1} 1_{I_{i j}^{n}},
$$

then

$$
\begin{aligned}
& \rho_{n}(r, z) \rightarrow \rho(r, z)=r, \quad \text { in } L^{q}\left(\mathbb{R}^{2}, \mathbb{P}\right), \\
& \sigma_{n}(r, z) \rightarrow \sigma(r, z)=z, \quad \text { in } L^{\infty}\left(\mathbb{R}^{2}, \mathbb{P}\right) .
\end{aligned}
$$

Combining the above ingredients, for $n \in \mathbb{N}$, we consider the following discretized variational inequality: find $\hat{u}_{n} \in X_{n} \cap C_{\mathbb{P}}$ such that for every $v_{n} \in X_{n} \cap C_{\mathbb{P}}$, we have

$$
\int_{0}^{\infty} \int_{\underline{z}}^{\bar{z}}\left\langle\sigma_{n}(y) G\left(\hat{u}_{n}\right)+H\left(\hat{u}_{n}\right), v_{n}-\hat{u}_{n}\right\rangle d \mathbb{P}(y) \geq \int_{0}^{\infty} \int_{\underline{z}}^{\bar{z}}\left\langle b+\rho_{n}(y) c, v_{n}-\hat{u}_{n}\right\rangle d \mathbb{P}(y) .
$$

The above approximated variational inequalities can be split in a finite number of finite dimensional variational inequalities, one for each interval $I_{i j}^{n}$, that is for every $n \in \mathbb{N}$ and for every $i, j$ find $\hat{u}_{i j}^{n} \in C$ such that

$$
\left\langle z_{n}^{j-1} G\left(\hat{u}_{i j}^{n}\right)+H\left(\hat{u}_{i j}^{n}\right), v_{i j}^{n}-\hat{u}_{i j}^{n}\right\rangle \geq\left\langle b+r_{n}^{i-1} c, v_{i j}^{n}-\hat{u}_{i j}^{n}\right\rangle, \quad \forall v_{i j}^{n} \in C .
$$

Clearly, we can reconstruct the solutions $\hat{u}_{n}$ as follows:

$$
\hat{u}_{n}=\sum_{i=1}^{N_{n}^{R}} \sum_{j=1}^{N_{n}^{Z}} \hat{u}_{i j}^{n} 1_{I_{i j}^{n}} \in X_{n} .
$$

We recall the following convergence result (see [15]).

Theorem 3.1. If $F(\omega, \cdot)$ is strongly monotone uniformly with respect to $\omega \in \Omega$ and the growth condition (12) holds, then the sequence $\left\{\hat{u}_{n}\right\}$ converges strongly in $L^{p}\left(\mathbb{R}^{2}, \mathbb{P}, \mathbb{R}^{N}\right)$ to the unique solution $\hat{u}$ of (15).

A consequence of the norm convergence stated in Theorem 3.1 is that both the mean value and variance of the solution $\hat{u}$ of (15) can be approximated through convergent sequences. The knowledge of the mean value of the equilibrium solution has clear practical implications for all the decision makers involved in the supply chain (generators, suppliers and consumers). However, also the knowledge of the variance of the equilibrium solution can provide useful information (e.g. to power generators and suppliers). In fact, it is well known that electric power cannot be easily and efficiently stored and the purchased electricity must be completely used or wasted so as to keep the distribution grid balanced. Thus, both shortage and surplus may cause an additional cost: the former one because the missing electricity must be acquired when needed, at a price usually greater than in the day ahead market, the latter because the electricity must be sold at a price usually smaller than the purchase price, or, even worse, wasted. Therefore, the variance of the equilibrium solution provides significant insights on the risk of possible additional cost the producers and suppliers may incur in and may be helpful in planning recovery strategies. 


\section{Monotonicity properties}

Since the theory of random variational inequalities outlined in the previous section is based on the monotonicity properties of the operator, in this section we give sufficient conditions to ensure the (strong) monotonicity of the map $F$ defined in (7). We introduce three maps: $A: \mathbb{R}^{G S} \rightarrow \mathbb{R}^{G S}$ defined as

$$
A_{g s}\left(Q^{1}\right)=\frac{\partial f_{g}\left(Q^{1}\right)}{\partial Q_{g s}^{1}}+\frac{\partial c_{g s}\left(Q^{1}\right)}{\partial Q_{g s}^{1}}-\rho_{g s}^{1},
$$

$B: \mathbb{R}^{G S+S K T} \rightarrow \mathbb{R}^{G S+S K T}$ defined as

$$
B\left(Q^{1}, Q^{2}\right)=\left(B^{1}\left(Q^{1}, Q^{2}\right), B^{2}\left(Q^{1}, Q^{2}\right)\right),
$$

where

$$
\begin{gathered}
B_{g s}^{1}\left(Q^{1}, Q^{2}\right)=\frac{\partial c_{s}\left(Q^{1}, Q^{2}\right)}{\partial Q_{g s}^{1}}+\frac{\partial \hat{c}_{g s}\left(Q^{1}\right)}{\partial Q_{g s}^{1}}+\rho_{g s}^{1}, \\
B_{s k t}^{2}\left(Q^{1}, Q^{2}\right)=\frac{\partial c_{s}\left(Q^{1}, Q^{2}\right)}{\partial Q_{s k t}^{2}}+\frac{\partial c_{s k t}\left(Q^{2}\right)}{\partial Q_{s k t}^{2}}-\rho_{s k t}^{2},
\end{gathered}
$$

and $D: \Omega \times \mathbb{R}^{S K T+K} \rightarrow \mathbb{R}^{S K T+K}$ defined as

$$
D\left(\omega, Q^{2}, \rho^{3}\right)=\left(D^{2}\left(Q^{2}, \rho^{3}\right), D^{3}\left(\omega, Q^{2}, \rho^{3}\right)\right)
$$

where

$$
D_{s k t}^{2}\left(Q^{2}, \rho^{3}\right)=\rho_{s k t}^{2}+\hat{c}_{s k t}\left(Q^{2}\right)-\rho_{k}^{3}, \quad D_{k}^{3}\left(\omega, Q^{2}, \rho^{3}\right)=\sum_{s=1}^{S} \sum_{t=1}^{T} Q_{s k t}^{2}-d_{k}\left(\omega, \rho^{3}\right) .
$$

Notice that $\rho^{1}$ and $\rho^{2}$ have the role of parameters in the definition of the above three maps. Then, it is easy to check that

$$
F^{1}=A+B^{1}, \quad F^{2}=B^{2}+D^{2}, \quad F^{3}=D^{3} .
$$

\section{Theorem 4.1.}

a) If $A, B$ and $D(\omega, \cdot)$ are monotone, then $F(\omega, \cdot)$ is monotone;

b) If $D(\omega, \cdot)$ is strongly monotone uniformly with respect to $\omega \in \Omega$, one out of $A$ or $B$ is strongly monotone while the other is monotone, then $F(\omega, \cdot)$ is strongly monotone uniformly with respect to $\omega \in \Omega$. 
Proof. Let $x=\left(Q^{1}, Q^{2}, \rho^{3}\right)$ and $\widetilde{x}=\left(\widetilde{Q}^{1}, \widetilde{Q}^{2}, \widetilde{\rho}^{3}\right)$. Then the following equalities hold:

$$
\begin{aligned}
\langle F(\omega, x)-F(\omega, \widetilde{x}), x-\widetilde{x}\rangle= & \left\langle F^{1}(x)-F^{1}(\widetilde{x}), Q^{1}-\widetilde{Q}^{1}\right\rangle \\
& +\left\langle F^{2}(x)-F^{2}(\widetilde{x}), Q^{2}-\widetilde{Q}^{2}\right\rangle \\
& +\left\langle F^{3}(\omega, x)-F^{3}(\omega, \widetilde{x}), \rho^{3}-\widetilde{\rho}^{3}\right\rangle \\
= & \left\langle A\left(Q^{1}\right)-A\left(\widetilde{Q}^{1}\right)+B^{1}\left(Q^{1}, Q^{2}\right)-B^{1}\left(\widetilde{Q}^{1}, \widetilde{Q}^{2}\right), Q^{1}-\widetilde{Q}^{1}\right\rangle \\
& +\left\langle B^{2}\left(Q^{1}, Q^{2}\right)-B^{2}\left(\widetilde{Q}^{1}, \widetilde{Q}^{2}\right)+D^{2}\left(Q^{2}, \rho^{3}\right)-D^{2}\left(\widetilde{Q}^{2}, \widetilde{\rho}^{3}\right), Q^{2}-\widetilde{Q}^{2}\right\rangle \\
& +\left\langle D^{3}\left(\omega, Q^{2}, \rho^{3}\right)-D^{3}\left(\omega, \widetilde{Q}^{2}, \widetilde{\rho}^{3}\right), \rho^{3}-\widetilde{\rho}^{3}\right\rangle \\
= & \left\langle A\left(Q^{1}\right)-A\left(\widetilde{Q}^{1}\right), Q^{1}-\widetilde{Q}^{1}\right\rangle \\
& +\left\langle B\left(Q^{1}, Q^{2}\right)-B\left(\widetilde{Q}^{1}, \widetilde{Q}^{2}\right),\left(Q^{1}, Q^{2}\right)-\left(\widetilde{Q}^{1}, \widetilde{Q}^{2}\right)\right\rangle \\
& \left.+D\left(\omega, Q^{2}, \rho^{3}\right)-D\left(\omega, \widetilde{Q}^{2}, \widetilde{\rho}^{3}\right),\left(Q^{2}, \rho^{3}\right)-\left(\widetilde{Q}^{2}, \widetilde{\rho}^{3}\right)\right\rangle
\end{aligned}
$$

a) It follows directly from the above equalities.

b) Assume that $D(\omega, \cdot)$ is strongly monotone uniformly with respect to $\omega \in \Omega$ with constant $\alpha_{D}$. If $A$ is strongly monotone with constant $\alpha_{A}$ and $B$ is monotone, then we have

$$
\begin{aligned}
\langle F(\omega, x)-F(\omega, \widetilde{x}), x-\widetilde{x}\rangle & \geq \alpha_{A}\left\|Q^{1}-\widetilde{Q}^{1}\right\|^{2}+\alpha_{D}\left\|\left(Q^{2}, \rho^{3}\right)-\left(\widetilde{Q}^{2}, \widetilde{\rho}^{3}\right)\right\|^{2} \\
& \geq \min \left\{\alpha_{A}, \alpha_{D}\right\}\|x-\widetilde{x}\|^{2}
\end{aligned}
$$

that is $F(\omega, \cdot)$ is strongly monotone uniformly with respect to $\omega \in \Omega$ with constant $\min \left\{\alpha_{A}, \alpha_{D}\right\}$. Similarly, if $A$ is monotone and $B$ is strongly monotone with constant $\alpha_{B}$, we have

$$
\begin{aligned}
\langle F(\omega, x)-F(\omega, \widetilde{x}), x-\widetilde{x}\rangle & \geq \alpha_{B}\left\|\left(Q^{1}, Q^{2}\right)-\left(\widetilde{Q}^{1}, \widetilde{Q}^{2}\right)\right\|^{2}+\alpha_{D}\left\|\left(Q^{2}, \rho^{3}\right)-\left(\widetilde{Q}^{2}, \widetilde{\rho}^{3}\right)\right\|^{2} \\
& \geq \min \left\{\alpha_{B}, \alpha_{D}\right\}\|x-\widetilde{x}\|^{2},
\end{aligned}
$$

that is $F(\omega, \cdot)$ is strongly monotone uniformly with respect to $\omega \in \Omega$ with constant $\min \left\{\alpha_{B}, \alpha_{D}\right\}$.

\section{The affine case}

In most applications the cost functions are quadratic and the demand functions are affine, thus yielding to an affine variational inequality. In this case, it is important to verify under which assumptions the monotonicity properties of $F$ investigated in Section 4 are fulfilled. In what follows, after specifying the functional form of all the cost and demand functions, we derive the operators $A, B$ and $D$ introduced previously and analyze their matrix structure. We recall that for an affine operator the strong monotonicity is equivalent to the positive definiteness of its Jacobian matrix. Moreover, the boundedness of $z=Z(\omega)$ allows proving that the strong monotonicity is uniform with respect to the random variable $\omega$. 
Theorem 5.1. Assume that, for any $g=1, \ldots, G, s=1, \ldots, S, k=1, \ldots, K, t=1, \ldots, T$, the cost and demand functions are defined as follows:

$$
\left\{\begin{array}{rlrl}
f_{g}\left(Q^{1}\right) & =\frac{1}{2}\left(Q^{1}\right)^{T} \Phi^{g} Q^{1}+\left(\varphi^{g}\right)^{T} Q^{1} & & \text { with } \Phi^{g} \in \mathbb{R}^{G S \times G S}, \varphi^{g} \in \mathbb{R}^{G S}, \\
c_{g s}\left(Q^{1}\right) & =\frac{1}{2} \gamma_{g s}\left(Q_{g s}^{1}\right)^{2}+\gamma_{g s}^{\prime} Q_{g s}^{1} & & \text { with } \gamma_{g s} \geq 0, \gamma_{g s}^{\prime} \in \mathbb{R}, \\
c_{s}\left(Q^{1}\right) & =\frac{1}{2} \gamma_{s}\left(\sum_{g=1}^{G} Q_{g s}^{1}\right)^{2} & & \text { with } \gamma_{s}>0, \\
\hat{c}_{g s}\left(Q^{1}\right) & =\frac{1}{2} \hat{\gamma}_{g s}\left(Q_{g s}^{1}\right)^{2}+\hat{\gamma}_{g s}^{\prime} Q_{g s}^{1} & & \text { with } \hat{\gamma}_{g s} \geq 0, \hat{\gamma}_{g s}^{\prime} \in \mathbb{R}, \\
c_{s k t}\left(Q^{2}\right) & =\frac{1}{2} \gamma_{s k t}\left(Q_{s k t}^{2}\right)^{2}+\gamma_{s k t}^{\prime} Q_{s k t}^{2} & & \text { with } \gamma_{s k t} \geq 0, \gamma_{s k t}^{\prime} \in \mathbb{R}, \\
\hat{c}_{s k t}\left(Q^{2}\right) & =\left(\hat{\Gamma} Q^{2}+\hat{\gamma}\right)_{s k t} & \text { with } \hat{\Gamma} \in \mathbb{R}^{S K T \times S K T}, \hat{\gamma} \in \mathbb{R}{ }^{S K T}, \\
d\left(\omega, \rho^{3}\right) & =z \Delta \rho^{3}+\delta+r c & \text { with } \Delta \in \mathbb{R}^{K \times K}, \delta, c \in \mathbb{R}^{K}, r, z \in \mathbb{R} .
\end{array}\right.
$$

where, in the last line, $r=R(\omega)$ and $z=Z(\omega)$ are two random variables, with $0<\underline{z}<z<\bar{z}$. Then, the following statements hold:

a) The maps $A, B, D(\omega, \cdot)$ and $F(\omega, \cdot)$ are affine for any $\omega \in \Omega$.

b) If the scalars $\gamma_{g s}$ are sufficiently large, then $A$ is a strongly monotone.

c) $B$ is monotone. Moreover, if $\hat{\gamma}_{g s}$ and $\gamma_{s k t}$ are sufficiently large, then $B$ is strongly monotone.

d) If $T=1, \hat{\Gamma}$ is positive definite and $\Delta$ is negative definite, then $D(\omega, \cdot)$ is strongly monotone uniformly with respect to $\omega \in \Omega$.

Proof.

a) For any $g=1, \ldots, G$ the matrix $\Phi^{g}$ and the vector $\varphi^{g}$ can be written as

$$
\Phi^{g}=\left(\begin{array}{c}
\Phi_{1}^{g} \\
\hline \Phi_{2}^{g} \\
\hline \vdots \\
\hline \Phi_{G}^{g}
\end{array}\right), \quad \varphi^{g}=\left(\begin{array}{c}
\varphi_{1}^{g} \\
\hline \varphi_{2}^{g} \\
\hline \vdots \\
\hline \varphi_{G}^{g}
\end{array}\right), \quad \text { where } \Phi_{i}^{g} \in \mathbb{R}^{S \times G S}, \quad \varphi_{i}^{g} \in \mathbb{R}^{S}, \quad \forall i=1, \ldots, G .
$$

Then, the map $A$ is affine with $A\left(Q^{1}\right)=\widetilde{A} Q^{1}+a$, where

$$
\widetilde{A}=\left(\begin{array}{c}
\Phi_{1}^{1} \\
\hline \Phi_{2}^{2} \\
\hline \vdots \\
\hline \Phi_{G}^{G}
\end{array}\right)+\left(\begin{array}{ccc}
\gamma_{11} & & 0 \\
& \ddots & \\
0 & & \gamma_{G S}
\end{array}\right), \quad a=\left(\begin{array}{c}
\varphi_{1}^{1} \\
\varphi_{2}^{2} \\
\hline \vdots \\
\hline \varphi_{G}^{G}
\end{array}\right)+\left(\begin{array}{c}
\gamma_{11}^{\prime} \\
\vdots \\
\gamma_{G S}^{\prime}
\end{array}\right)-\rho^{1}
$$


The map $B$ is affine with

$$
B\left(Q^{1}, Q^{2}\right)=\left(\begin{array}{c|c}
\widetilde{B}^{1} & 0 \\
\hline 0 & \widetilde{B}^{2}
\end{array}\right)\left(\begin{array}{c}
Q^{1} \\
\hline Q^{2}
\end{array}\right)+\left(\begin{array}{c}
b^{1} \\
\hline b^{2}
\end{array}\right)
$$

where

$$
\begin{aligned}
& \widetilde{B}^{1}=\left(\begin{array}{c|c|c}
\Gamma & \ldots & \Gamma \\
\hline \vdots & \ddots & \vdots \\
\hline \Gamma & \ldots & \Gamma
\end{array}\right)+\left(\begin{array}{ccc}
\hat{\gamma}_{11} & & 0 \\
& \ddots & \\
0 & & \hat{\gamma}_{G S}
\end{array}\right), \quad \text { with } \quad \Gamma=\left(\begin{array}{ccc}
\gamma_{1} & & 0 \\
& \ddots & \\
0 & & \gamma_{S}
\end{array}\right) \\
& \widetilde{B}^{2}=\left(\begin{array}{ccc}
\gamma_{111} & & 0 \\
& \ddots & \\
0 & & \gamma_{S K T}
\end{array}\right), \quad b^{1}=\left(\begin{array}{c}
\hat{\gamma}_{11}^{\prime} \\
\vdots \\
\hat{\gamma}_{G S}^{\prime}
\end{array}\right)+\rho^{1}, \quad b^{2}=\left(\begin{array}{c}
\gamma_{111}^{\prime} \\
\vdots \\
\gamma_{S K T}^{\prime}
\end{array}\right)-\rho^{2} .
\end{aligned}
$$

Finally, for any $\omega \in \Omega$, the map $D(\omega, \cdot)$ is affine with

$$
D\left(\omega, Q^{2}, \rho^{3}\right)=\widetilde{D}\left(\begin{array}{c}
Q^{2} \\
\hline \rho^{3}
\end{array}\right)+\left(\begin{array}{c}
\hat{\gamma}+\rho^{2} \\
\hline-\delta-R(\omega) c
\end{array}\right), \quad \text { where } \quad \widetilde{D}=\left(\begin{array}{c|c}
\hat{\Gamma} & D_{23} \\
\hline D_{32} & -Z(\omega) \Delta
\end{array}\right),
$$

where $D_{23}$ and $D_{32}$ are suitable matrices with 0,1 or -1 entries.

Therefore, it follows from (17) that also the map $F(\omega, \cdot)$ is affine for any $\omega$.

b) If the positive scalars $\gamma_{g s}$ are given sufficiently large, then the matrix $\left(\tilde{A}+\tilde{A}^{T}\right) / 2$ is diagonal dominant and positive definite, thus the map $A$ is strongly monotone.

c) It is easy to check that the matrices $\widetilde{B}^{1}$ and $\widetilde{B}^{2}$ are positive semidefinite, thus the map $B$ is monotone. Furthermore, if the scalars $\hat{\gamma}_{g s}$ and $\gamma_{s k t}$ are given sufficiently large, then $\widetilde{B}^{1}$ and $\widetilde{B}^{2}$ are diagonal dominant and positive definite, thus $B$ is strongly monotone.

d) If $T=1$, then the matrices $D_{23}$ and $D_{32}$ have a special structure, that is

$$
D_{23}=\left(\begin{array}{c}
-I_{K \times K} \\
\vdots \\
-I_{K \times K}
\end{array}\right)=-D_{32}^{T}
$$

where $I_{K \times K}$ is the identity matrix of order $K$. Therefore the matrix

$$
\frac{\widetilde{D}+\widetilde{D}^{T}}{2}=\left(\begin{array}{c|c}
\left(\hat{\Gamma}+\hat{\Gamma}^{T}\right) / 2 & 0 \\
\hline 0 & -Z(\omega)\left(\Delta+\Delta^{T}\right) / 2
\end{array}\right)
$$

Since $\hat{\Gamma}$ is positive definite, $\Delta$ is negative definite and $Z(\omega)>\underline{z}>0$, there exists $\alpha>0$ such that the minimum eigenvalue of $\left(\widetilde{D}+\widetilde{D}^{T}\right) / 2$ is grater or equal to $\alpha$ for any $\omega \in \Omega$. Therefore, the map $D(\omega, \cdot)$ is strongly monotone with constant $\alpha$ uniformly with respect to $\omega \in \Omega$. 


\section{Numerical experiments}

We now report some numerical results for the stochastic network equilibrium model by exploiting the approximation procedure described in Section 3. The approximation procedure has been implemented in MATLAB R2018a and tested on an Intel Core i7 system at $2.5 \mathrm{GHz}$ with 16 GB of RAM running under macOS 10.13. At each iteration $n$ a finite dimensional variational inequality as (16) has to be solved for any interval $I_{i j}^{n}$. Since any of these problems has an affine and strongly monotone map, it has been reformulated as an equivalent convex quadratic optimization problem (see [2]) and solved by means of the built-in function quadprog from the optimization toolbox.

In the following, Section 6.1 considers two instances of the problem with three power generators, two power suppliers, three demand markets and a single transmission service provider for each supplier. The convergence of the mean values and the standard deviations of the approximated solutions, according to Theorem 3.1, are shown. Section 6.2 shows the impact of different probability densities of the random variables $R$ and $Z$ on the mean values and the standard deviations of the approximated solutions. Finally, Section 6.3 shows the scalability of the numerical approximation procedure for real-world sized problems.

\subsection{Convergence of mean values and standard deviations of the approxi- mated solutions}

We consider two examples with three power generators, two power suppliers, three demand markets and a single transmission service provider for each supplier, that is $G=3, S=2$, $K=3$ and $T=1$. The cost and demand functions, inspired by [22], are defined as in (18) so that the map $F$ can be written in the form (10) and $F(\omega, \cdot)$ is affine for any $\omega \in \Omega$. Moreover, it is easy to check that the map $F(\omega, \cdot)$ is strongly monotone uniformly with respect to $\omega$, hence the convergence of the approximation procedure is guaranteed by Theorem 3.1.

Example 1. The cost and demand functions are defined as follows:

$$
\begin{aligned}
& f_{1}=5\left(Q_{11}^{1}+Q_{12}^{1}\right)^{2}+\left(Q_{11}^{1}+Q_{12}^{1}\right)\left(Q_{21}^{1}+Q_{22}^{1}\right)+2\left(Q_{11}^{1}+Q_{12}^{1}\right) \\
& f_{2}=2.5\left(Q_{21}^{1}+Q_{22}^{1}\right)^{2}+\left(Q_{11}^{1}+Q_{12}^{1}\right)\left(Q_{21}^{1}+Q_{22}^{1}\right)+2\left(Q_{21}^{1}+Q_{22}^{1}\right) \\
& f_{3}=0.5\left(Q_{31}^{1}+Q_{32}^{1}\right)^{2}+0.5\left(Q_{11}^{1}+Q_{12}^{1}\right)\left(Q_{31}^{1}+Q_{32}^{1}\right)+2\left(Q_{31}^{1}+Q_{32}^{1}\right) \\
& c_{11}=0.5\left(Q_{11}^{1}\right)^{2}+3.5 Q_{11}^{1} \\
& c_{12}=0.5\left(Q_{12}^{1}\right)^{2}+3.5 Q_{12}^{1} \\
& c_{21}=0.5\left(Q_{21}^{1}\right)^{2}+3.5 Q_{21}^{1} \\
& c_{22}=0.5\left(Q_{22}^{1}\right)^{2}+3.5 Q_{22}^{1} \\
& c_{31}=0.5\left(Q_{31}^{1}\right)^{2}+2 Q_{31}^{1} \\
& c_{32}=0.5\left(Q_{32}^{1}\right)^{2}+2 Q_{32}^{1} \\
& c_{1}=0.5\left(Q_{11}^{1}+Q_{21}^{1}+Q_{31}^{1}\right)^{2} \\
& c_{2}=0.5\left(Q_{12}^{1}+Q_{22}^{1}+Q_{32}^{1}\right)^{2} \\
& \hat{c}_{g s}=0, \quad \forall g=1,2,3, \forall s=1,2 \\
& c_{s k 1}=0, \quad \forall s=1,2, \forall k=1,2,3 \\
& \hat{c}_{s k 1}=Q_{s k 1}^{2}+5, \quad \forall s=1,2, \forall k=1,2,3 \\
& d_{1}=-2 z \rho_{1}^{3}+1500+r \\
& d_{2}=-2 z \rho_{2}^{3}+1100+r \\
& d_{3}=-2 z \rho_{3}^{3}+1200+r
\end{aligned}
$$

where $z=Z(\omega)$ and $r=R(\omega)$ are two random variables. We assume that $z$ is uniformly 
distributed in the interval $[0.5,1.5]$ and $r$ is uniformly distributed in $[-100,100]$. At each iteration, each of the two intervals has been partitioned into $N^{d}$ subintervals. Tables 1 and 2 show the convergence of the mean values and the standard deviations of the approximate solution obtained for different values of $N^{d}$, respectively.

Table 1: Mean values of the approximated solution of Example 1.

\begin{tabular}{|c|c|c|c|c|c|c|c|}
\hline \multirow[b]{2}{*}{ Variables } & \multicolumn{7}{|c|}{$N^{d}$} \\
\hline & 16 & 32 & 64 & 128 & 256 & 512 & 1024 \\
\hline$Q_{11}^{1}$ & 15.79 & 15.56 & 15.45 & 15.39 & 15.37 & 15.35 & 15.35 \\
\hline$Q_{12}^{1}$ & 15.79 & 15.56 & 15.45 & 15.39 & 15.37 & 15.35 & 15.35 \\
\hline$Q_{21}^{1}$ & 33.34 & 32.86 & 32.62 & 32.50 & 32.44 & 32.41 & 32.40 \\
\hline$Q_{22}^{1}$ & 33.34 & 32.86 & 32.62 & 32.50 & 32.44 & 32.41 & 32.40 \\
\hline$Q_{31}^{1}$ & 128.02 & 126.16 & 125.25 & 124.79 & 124.57 & 124.45 & 124.40 \\
\hline$Q_{32}^{1}$ & 128.02 & 126.16 & 125.25 & 124.79 & 124.57 & 124.45 & 124.40 \\
\hline $\bar{Q}_{11}^{2}$ & $\overline{1} 19.63$ & $1 \overline{1} 8.28$ & $1 \overline{1} \overline{7} . \overline{6} 1$ & $\overline{1} 1 \overline{7} . \overline{8}$ & $\overline{1} \overline{17} . \overline{11}$ & $1 \overline{1} \overline{7} . \overline{0} 3$ & $11 \overline{6} . \overline{9}$ \\
\hline$Q_{12}^{2}$ & 15.78 & 15.28 & 15.03 & 14.91 & 14.85 & 14.82 & 14.80 \\
\hline$Q_{13}^{2}$ & 41.74 & 41.03 & 40.67 & 40.50 & 40.41 & 40.37 & 40.35 \\
\hline$Q_{21}^{2}$ & 119.63 & 118.28 & 117.61 & 117.28 & 117.11 & 117.03 & 116.99 \\
\hline$Q_{22}^{2}$ & 15.78 & 15.28 & 15.03 & 14.91 & 14.85 & 14.82 & 14.80 \\
\hline$Q_{23}^{2}$ & 41.74 & 41.03 & 40.67 & 40.50 & 40.41 & 40.37 & 40.35 \\
\hline$\rho_{1}^{3}$ & $\overline{7} 0 \overline{5} . \overline{65}$ & $\overline{6} \overline{9} . \overline{9} 1$ & $69 \overline{1} . \overline{1} 2$ & $\overline{688} \overline{7} \overline{4}$ & $\overline{6} \overline{8} \overline{5} . \overline{56}$ & $6 \overline{8} \overline{6} . \overline{9} 6$ & $\overline{6} 8 \overline{6} . \overline{6} \overline{7}$ \\
\hline$\rho_{2}^{3}$ & 601.80 & 592.91 & 588.53 & 586.37 & 585.29 & 584.75 & 584.48 \\
\hline$\rho_{3}^{\overline{3}}$ & 627.77 & 618.66 & 614.18 & 611.96 & 610.85 & 610.30 & 610.03 \\
\hline
\end{tabular}

Example 2. The data of Example 2 are the same of Example 1 except the demand functions which are defined as

$$
\begin{aligned}
& d_{1}=-2 z \rho_{1}^{3}-1.5 z \rho_{2}^{3}+1500+r \\
& d_{2}=-1.5 z \rho_{1}^{3}-2 z \rho_{2}^{3}+1100+r \\
& d_{3}=-1.5 z \rho_{1}^{3}-2 z \rho_{3}^{3}+1200+r
\end{aligned}
$$

where $z$ is uniformly distributed in $[0.5,1.5]$ and $r$ is uniformly distributed in $[-100,100]$. Tables 3 and 4 show the convergence of the mean values and the standard deviations of the approximate solution obtained for different values of $N^{d}$, respectively. Moreover, we remark that the mean values of variables $Q_{12}^{2}, Q_{13}^{2}, Q_{22}^{2}, Q_{23}^{2}$ are close to zero since the equilibrium prices $\rho_{2}^{3}$ and $\rho_{3}^{3}$ of the demand markets 2 and 3 are very lower than the price $\rho_{1}^{3}$ associated with market 1 .

\subsection{The impact of the probability densities of the random parameters on the solution}

We now show how much the (approximated) solution of the stochastic variational inequality is sensitive to the choice of the probability densities of the random variables $z$ and $r$. We solved 
Table 2: Standard deviations of the approximated solution of Example 1.

\begin{tabular}{|c|c|c|c|c|c|c|c|}
\hline \multirow[b]{2}{*}{ Variables } & \multicolumn{7}{|c|}{$N^{d}$} \\
\hline & 16 & 32 & 64 & 128 & 256 & 512 & 1024 \\
\hline$Q_{11}^{1}$ & 4.87 & 4.73 & 4.66 & 4.62 & 4.61 & 4.60 & 4.59 \\
\hline$Q_{12}^{1}$ & 4.87 & 4.73 & 4.66 & 4.62 & 4.61 & 4.60 & 4.59 \\
\hline$Q_{21}^{1}$ & 10.28 & 9.99 & 9.84 & 9.76 & 9.72 & 9.71 & 9.70 \\
\hline$Q_{22}^{1}$ & 10.28 & 9.99 & 9.84 & 9.76 & 9.72 & 9.71 & 9.70 \\
\hline$Q_{31}^{1}$ & 39.32 & 38.20 & 37.62 & 37.34 & 37.19 & 37.12 & 37.08 \\
\hline$Q_{32}^{1}$ & 39.32 & 38.20 & 37.62 & 37.34 & 37.19 & 37.12 & 37.08 \\
\hline $\bar{Q}_{11}^{2}$ & $\overline{27.1 \overline{3}}$ & $\overline{2} \overline{6} . \overline{4} 8$ & $\overline{2} \overline{6} . \overline{1} \overline{5}$ & $\overline{25.98}$ & $\overline{2} 5 . \overline{90}$ & $\overline{2} \overline{5} . \overline{8} 6$ & $2 \overline{5} . \overline{8} \overline{4}$ \\
\hline$Q_{12}^{2}$ & 11.86 & 11.44 & 11.22 & 11.12 & 11.06 & 11.03 & 11.02 \\
\hline$Q_{13}^{2}$ & 15.62 & 15.14 & 14.89 & 14.77 & 14.71 & 14.68 & 14.66 \\
\hline$Q_{21}^{2}$ & 27.13 & 26.48 & 26.15 & 25.98 & 25.90 & 25.86 & 25.84 \\
\hline$Q_{22}^{2}$ & 11.86 & 11.44 & 11.22 & 11.12 & 11.06 & 11.03 & 11.02 \\
\hline$Q_{23}^{2}$ & 15.62 & 15.14 & 14.89 & 14.77 & 14.71 & 14.68 & 14.66 \\
\hline$\rho_{1}^{3}$ & $204.3 \overline{7}$ & $\overline{19} 8 . \overline{6} 6$ & $19 \overline{5} . \overline{7} \overline{5}$ & 194.29 & $\overline{193.55}$ & $\overline{19} . \overline{19}$ & $\overline{193} . \overline{0}$ \\
\hline$\rho_{2}^{3}$ & 189.09 & 183.60 & 180.81 & 179.41 & 178.70 & 178.35 & 178.17 \\
\hline$\rho_{3}^{3}$ & 192.90 & 187.36 & 184.54 & 183.12 & 182.41 & 182.05 & 181.88 \\
\hline
\end{tabular}

Table 3: Mean values of the approximated solution of Example 2.

\begin{tabular}{|c|c|c|c|c|c|c|c|}
\hline \multirow[b]{2}{*}{ Variables } & \multicolumn{7}{|c|}{$N^{d}$} \\
\hline & 16 & 32 & 64 & 128 & 256 & 512 & 1024 \\
\hline$Q_{11}^{1}$ & 11.03 & 10.91 & 10.85 & 10.82 & 10.81 & 10.80 & 10.79 \\
\hline$Q_{12}^{1}$ & 11.03 & 10.91 & 10.85 & 10.82 & 10.81 & 10.80 & 10.79 \\
\hline$Q_{21}^{1}$ & 23.28 & 23.03 & 22.90 & 22.84 & 22.81 & 22.80 & 22.79 \\
\hline$Q_{22}^{1}$ & 23.28 & 23.03 & 22.90 & 22.84 & 22.81 & 22.80 & 22.79 \\
\hline$Q_{31}^{1}$ & 89.55 & 88.58 & 88.10 & 87.86 & 87.75 & 87.69 & 87.66 \\
\hline$Q_{32}^{1}$ & 89.55 & 88.58 & 88.10 & 87.86 & 87.75 & 87.69 & 87.66 \\
\hline $\bar{Q}_{11}^{2}$ & $\overline{123.18}$ & $\overline{1} \overline{2} 2.0 \overline{1}$ & $\overline{12} \overline{1} . \overline{4} 3$ & $\overline{1} 2 \overline{1} . \overline{1} \overline{4}$ & $\overline{1} \overline{2} 1 . \overline{0}-\overline{ }$ & $\overline{12} \overline{0} . \overline{9} 2$ & $\overline{1} 2 \overline{0} . \overline{8} \overline{9}$ \\
\hline$Q_{12}^{2}$ & 0.00 & 0.00 & 0.00 & 0.00 & 0.00 & 0.00 & 0.00 \\
\hline$Q_{13}^{2}$ & 0.68 & 0.50 & 0.42 & 0.39 & 0.37 & 0.36 & 0.35 \\
\hline$Q_{21}^{2}$ & 123.18 & 122.01 & 121.43 & 121.14 & 121.00 & 120.92 & 120.89 \\
\hline$Q_{22}^{2}$ & 0.00 & 0.00 & 0.00 & 0.00 & 0.00 & 0.00 & 0.00 \\
\hline$Q_{23}^{2}$ & 0.68 & 0.50 & 0.42 & 0.39 & 0.37 & 0.36 & 0.35 \\
\hline$\rho_{1}^{3}$ & $\overline{5} 35.7 \overline{3}$ & 530.17 & $52 \overline{7} . \overline{4} \overline{4}$ & $\overline{5} 2 \overline{6} . \overline{8}$ & $\overline{5} 5 . \overline{4}$ & $5 \overline{2} \overline{5} . \overline{0} 7$ & 524.90 \\
\hline$\rho_{2}^{3}$ & 222.43 & 216.48 & 213.56 & 212.12 & 211.40 & 211.04 & 210.86 \\
\hline$\rho_{3}^{3}$ & 278.15 & 271.48 & 268.20 & 266.57 & 265.76 & 265.35 & 265.15 \\
\hline
\end{tabular}


Table 4: Standard deviations of the approximated solution of Example 2.

\begin{tabular}{|c|c|c|c|c|c|c|c|}
\hline \multirow[b]{2}{*}{ Variables } & \multicolumn{7}{|c|}{$N^{d}$} \\
\hline & 16 & 32 & 64 & 128 & 256 & 512 & 1024 \\
\hline$Q_{11}^{1}$ & 2.34 & 2.28 & 2.25 & 2.23 & 2.23 & 2.22 & 2.22 \\
\hline$Q_{12}^{1}$ & 2.34 & 2.28 & 2.25 & 2.23 & 2.23 & 2.22 & 2.22 \\
\hline$Q_{21}^{1}$ & 4.95 & 4.82 & 4.75 & 4.72 & 4.70 & 4.69 & 4.69 \\
\hline$Q_{22}^{1}$ & 4.95 & 4.82 & 4.75 & 4.72 & 4.70 & 4.69 & 4.69 \\
\hline$Q_{31}^{1}$ & 18.92 & 18.42 & 18.17 & 18.04 & 17.98 & 17.95 & 17.93 \\
\hline$Q_{32}^{1}$ & 18.92 & 18.42 & 18.17 & 18.04 & 17.98 & 17.95 & 17.93 \\
\hline $\bar{Q}_{11}^{2}$ & $24.8 \overline{1}$ & $\overline{2} \overline{4} . \overline{4} 5$ & $\overline{2} \overline{4} . \overline{2} \overline{6}$ & $24 . \overline{1} \overline{6}$ & $\overline{2} \overline{4} . \overline{11}$ & $\overline{2} \overline{4} . \overline{0} \overline{9}^{-}$ & $2 \overline{4} . \overline{0} \overline{8}$ \\
\hline$Q_{12}^{2}$ & 0.00 & 0.00 & 0.00 & 0.00 & 0.00 & 0.00 & 0.00 \\
\hline$Q_{13}^{2}$ & 2.71 & 2.26 & 2.02 & 1.90 & 1.84 & 1.81 & 1.79 \\
\hline$Q_{21}^{2}$ & 24.81 & 24.45 & 24.26 & 24.16 & 24.11 & 24.09 & 24.08 \\
\hline$Q_{22}^{2}$ & 0.00 & 0.00 & 0.00 & 0.00 & 0.00 & 0.00 & 0.00 \\
\hline$Q_{23}^{2}$ & 2.71 & 2.26 & 2.02 & 1.90 & 1.84 & 1.81 & 1.79 \\
\hline$\rho_{1}^{3}$ & $\overline{1} 10.06$ & $107 . \overline{4} 5$ & $10 \overline{6} . \overline{1}$ & $\overline{105.46}$ & 105.13 & 104.96 & $10 \overline{4} . \overline{8}$ \\
\hline$\rho_{2}^{3}$ & 130.43 & 125.53 & 123.05 & 121.80 & 121.17 & 120.86 & 120.70 \\
\hline$\rho_{3}^{\overline{3}}$ & 146.08 & 141.27 & 138.80 & 137.56 & 136.93 & 136.61 & 136.46 \\
\hline
\end{tabular}

Example 1 described in Section 6.1 considering three different probability densities: uniform, truncated normal and exponential. In particular, we assumed that $z$ varies in the interval $[0.5,1.5]$ with either uniform or truncated normal (with mean value 1 and standard deviation 0.125 ) distribution, while $r$ varies in the interval $[0,200]$ with either truncated normal (with mean value 100 and standard deviation 25) or exponential (with parameter equal to 0.1) distribution. The four different combinations of probability densities of $z$ and $r$ are denoted by U-N, U-E, N-N and N-E; for instance, U-N means that $z$ has a uniform distribution, while $r$ has a truncated normal distribution and so on. Both intervals $[0.5,1.5]$ and $[0,200]$ have been partitioned into 1024 subintervals in the approximation procedure. Tables 5 and 6 report the mean values and the standard deviations of the approximated solution of Example 1 obtained by using the four different combinations of probability densities.

As table 5 shows, the solutions can vary considerably from the first to the fourth column. For instance, $Q_{22}^{2}$ changes of about $36 \%$, while $\rho_{2}^{3}$ of about $23 \%$. We assume that these distributions are derived from collections of market's data in a given time window and can thus be used to analyze the average behavior of consumers and design future market strategies. We recall that the flows $Q^{1}$ and $Q^{2}$ in our model represent the financial transactions flows and the analysis of the mean values over a given time interval can provide information about how the various companies in competition react to the demand perturbations.

As to the analysis of standard deviations, which is missing, to the best of our knowledge, in most random models of electricity markets, we notice that, for a given pair of probability distributions, the ratio between the standard deviation and the corresponding mean value is approximately constant for all the variables. On the other hand, it can change considerably according to different distributions. Indeed, this value is about 0.30 for columns $2-3$ in 
Table 5: The impact of different probability densities on the mean values of the approximated solution of Example 1.

\begin{tabular}{|c|c|c|c|c|}
\hline ariables & $\overline{\mathrm{U}-\mathrm{N}}$ & U-E & $\mathrm{N}-\mathrm{N}$ & $\mathrm{N}-\mathrm{E}$ \\
\hline$Q_{11}^{1}$ & 16.58 & 15.47 & 15.54 & 14.50 \\
\hline$Q_{12}^{1}$ & 16.58 & 15.47 & 15.54 & 14.50 \\
\hline$Q_{21}^{1}$ & 35.00 & 32.65 & 32.80 & 30.61 \\
\hline$Q_{22}^{1}$ & 35.00 & 32.65 & 32.80 & 30.61 \\
\hline$Q_{31}^{1}$ & 134.34 & 125.39 & 125.96 & 117.55 \\
\hline$Q_{32}^{1}$ & 134.34 & 125.39 & 125.96 & 117.55 \\
\hline $\bar{Q}_{11}^{2}$ & $\overline{1} 2 \overline{1} . \overline{8}$ & $\overline{1} \overline{1} \overline{7} . \overline{45}$ & $1 \overline{1} \overline{6} . \overline{6} 8$ & $\overline{112} \overline{8} \overline{0}$ \\
\hline$Q_{12}^{2}$ & 19.39 & 15.26 & 16.26 & 12.38 \\
\hline$Q_{13}^{2}$ & 44.94 & 40.80 & 41.36 & 37.48 \\
\hline$Q_{21}^{2}$ & 121.58 & 117.45 & 116.68 & 112.80 \\
\hline$Q_{22}^{2}$ & 19.39 & 15.26 & 16.26 & 12.38 \\
\hline$Q_{23}^{2}$ & 44.94 & 40.80 & 41.36 & 37.48 \\
\hline$\rho_{1}^{3}$ & $\overline{7} \overline{36} . \overline{1}$ & $6 \overline{9} 1.59$ & $6 \overline{9} \overline{3} . \overline{3} 9$ & $\overline{6} 5 \overline{1} . \overline{6} \overline{1}$ \\
\hline$\rho_{2}^{3}$ & 633.92 & 589.39 & 592.98 & 551.19 \\
\hline$\rho_{3}^{3}$ & 659.47 & 614.94 & 618.08 & 576.30 \\
\hline
\end{tabular}

Table 6: The impact of different probability densities on the standard deviations of the approximated solution of Example 1.

\begin{tabular}{|c|c|c|c|c|}
\hline Variables & U-N & U-E & $\mathrm{N}-\mathrm{N}$ & $\mathrm{N}-\mathrm{E}$ \\
\hline$Q_{11}^{1}$ & 4.90 & 4.57 & 1.91 & 1.77 \\
\hline$Q_{12}^{1}$ & 4.90 & 4.57 & 1.91 & 1.77 \\
\hline$Q_{21}^{1}$ & 10.35 & 9.65 & 4.03 & 3.73 \\
\hline$Q_{22}^{1}$ & 10.35 & 9.65 & 4.03 & 3.73 \\
\hline$Q_{31}^{1}$ & 39.57 & 36.90 & 15.41 & 14.26 \\
\hline$Q_{32}^{1}$ & 39.57 & 36.90 & 15.41 & 14.26 \\
\hline $\bar{Q}_{11}^{2}$ & $2 \overline{7} . \overline{0} \overline{5}$ & $2 \overline{5} \overline{8} \overline{2}$ & $\overline{1} 0 . \overline{78}$ & $10 . \overline{2} \overline{8}$ \\
\hline$Q_{12}^{2}$ & 12.06 & 10.81 & 4.53 & 3.96 \\
\hline$Q_{13}^{2}$ & 15.78 & 14.54 & 6.08 & 5.53 \\
\hline$Q_{21}^{2}$ & 27.05 & 25.82 & 10.78 & 10.28 \\
\hline$Q_{22}^{2}$ & 12.06 & 10.81 & 4.53 & 3.96 \\
\hline$Q_{23}^{2}$ & 15.78 & 14.54 & 6.08 & 5.53 \\
\hline$\rho_{1}^{3}$ & $20 \overline{5} . \overline{4} \overline{5}$ & $\overline{192 . \overline{2}}$ & $\overline{8} 0 . \overline{2} 6$ & $\overline{4} \overline{4} . \overline{5}$ \\
\hline$\rho_{2}^{3}$ & 190.46 & 177.18 & 74.00 & 68.25 \\
\hline$\rho_{3}^{3}$ & 194.20 & 180.93 & 75.56 & 69.83 \\
\hline
\end{tabular}


Tables 5 and 6 , while it is about 0.10 for columns $4-5$. Higher values of standard deviations are associated to periods of strong volatility of markets, which are becoming more frequent, because of the ever increasing connections among the economies of different countries.

\subsection{Scalability of the proposed approach}

In this section we show how the proposed numerical method scales for real-world sized problems. We consider a set of random generated instances, where the number $G$ of power generators is between 3 and 8 , the number $S$ of power suppliers is between 2 and 25 (with a single transmission service provider for each supplier, i.e., $T=1$ ) and the number $K$ of demand markets is between 3 and 12 (see columns $1-3$ of Table 7 ). The cost and demand functions have been chosen as in (18), where the elements of matrices $\Phi^{g}$ are uniformly distributed in the interval $(0,1 / G S)$, the elements of vectors $\varphi^{g}$ are uniformly distributed in $(1,2), \gamma_{g s}$ and $\gamma_{g s}^{\prime}$ are uniformly distributed in $(2,3), \gamma_{s}=1$ for any $s, \hat{\gamma}_{g s}=\hat{\gamma}_{g s}^{\prime}=\gamma_{s k t}=\gamma_{s k t}^{\prime}=0$ for any $g, s, k, t$, the matrix $\hat{\Gamma}$ is equal to the identity matrix, $\hat{\gamma}_{s k t}=5$ for any $s, k, t$, the matrix $\Delta$ is defined as

$$
\Delta=-\left(\begin{array}{ccccc}
a_{1} & b_{1} & & & 0 \\
0 & \ddots & \ddots & & \\
& \ddots & \ddots & \ddots & \\
& & 0 & a_{K-1} & b_{K-1} \\
0 & & & b_{K} & a_{K}
\end{array}\right),
$$

where $a_{k}$ are uniformly distributed in $(1,2)$ and $b_{k}$ are uniformly distributed in $(0,1)$ for any $k=1, \ldots, K$; the elements of vector $\delta$ are uniformly distributed in $(1000,1300)$, while $c=(1, \ldots, 1)$. In this framework, it is possible to verify that the assumptions of Theorems 4.1 and 5.1 are satisfied, hence the map $F(\omega, \cdot)$ is strongly monotone uniformly with respect to $\omega$ and the convergence of the approximation procedure is guaranteed by Theorem 3.1. Finally, random variables $z$ and $r$ are uniformly distributed in the intervals $[0.5,1.5]$ and $[-100,100]$, respectively.

Table 7 shows the CPU times needed for solving the six random generated instances. In particular, columns 1-3 report the number of power generators, power suppliers and demand markets of each instance; column 4 reports the number of variables of the stochastic variational inequality to be solved; columns 5-7 report the CPU times (in seconds) of the numerical approximation procedure, where each interval of the random variables is divided in 64,128 or 256 subintervals.

The numerical results show that the proposed numerical method solves real-world sized problems with a good accuracy within satisfactory times. Moreover, we notice that the approximation procedure has been implemented using a sequential algorithm, that is the independent deterministic variational inequalities are solved one at a time. It is clear that parallel computing techniques could be used profitably to improve the running times.

\section{Conclusions}

In this paper, we propose a stochastic variational inequality model for the whole chain of electricity markets, from generators to consumers. In particular, our approach takes into account random perturbations of the consumers' demand. Monotonicity properties of the operator of 
Table 7: Scalability of the discretization procedure (CPU times in seconds).

\begin{tabular}{rrr|c|rrr}
\hline & & & \multicolumn{3}{|c}{$N^{d}$} \\
$G$ & $S$ & $K$ & \# variables & 64 & 128 & 256 \\
\hline 3 & 2 & 3 & 15 & 5.49 & 20.59 & 81.94 \\
4 & 5 & 4 & 44 & 10.06 & 40.21 & 160.68 \\
5 & 10 & 6 & 116 & 38.04 & 152.20 & 609.29 \\
6 & 15 & 8 & 218 & 115.49 & 462.00 & 1833.66 \\
7 & 20 & 10 & 350 & 346.00 & 1391.17 & 5586.22 \\
8 & 25 & 12 & 512 & 813.57 & 3232.08 & 12896.10 \\
\hline
\end{tabular}

the variational inequality and the case of quadratic cost and linear demand functions are investigated in detail. Numerical experiments show the impact of different probability densities of the random variables on the approximated solutions and the scalability of the numerical approximation procedure for real-world sized problems. Our approximation procedure for the stochastic variational inequality yields to a large number of deterministic and independent variational inequalities, hence through parallelization one could increase the number of independent random variables in the model. Moreover, the model could be further refined by including the description of the physical network, or the investment strategies (see [11]). These last points are beyond the scope of this paper and are left to future research.

\section{References}

[1] J. Abrell, F. Kunz, Integrating Intermittent Renewable Wind Generation - A Stochastic Multi-Market Electricity Model for the European Electricity Market, Networks and Spatial Economics 15 (2015), pp. 117-147.

[2] M. Aghassi, D. Bertsimas, G. Perakis, Solving asymmetric variational inequalities via convex optimization, Operations Research Letters 34 (2006), pp. 481-490.

[3] A. Banal-Estañol, A. Rupérez Micola, Behavioural simulations in spot electricity markets, European Journal of Operational Research 214 (2011), pp. 147-159.

[4] A. Causa, B. Jadamba, F. Raciti, A migration equilibrium model with uncertain data and movement costs, Decisions in Economics and Finance Volume 40 (2017), pp. 159-175.

[5] A.J. Conejo, M. Carrion, J.M. Morales, Decision Making Under Uncertainty in Electricity Markets, Springer, 2010.

[6] P. Couchman, B. Kouvaritakis, M. Cannon, F. Prashad, Gaming strategy for electric power with random demand, IEEE Transactions on Power Systems 20 (2005), pp. 11991206.

[7] O. Daxhelet, Y. Smeers, Variational inequality models of restructered electricity systems. In: M.C. Ferris, O.L. Mangasarian, J.-S. Pang (eds) Applications and algorithms of complementarity, Kluwer, Dordrecht, 2001. 
[8] R. Eldor, I. Zilcha, Oligopoly, uncertain demand, and forward markets, Journal of Economics and Business 42 (1990), pp. 17-26.

[9] F. Faraci, B. Jadamba, F. Raciti, On stochastic variational inequalities with mean value constraints, Journal of Optimization Theory and Applications 171 (2016), pp. 675-693.

[10] D. Gabay, H. Moulin, On the uniqueness and stability of Nash equilibria in Noncooperative games. In: Applied stochastic control in econometrics and management science, A. Bensoussan, P. Kleindorfer, C.S. Tapiero (eds), North-Holland Amsterdam, pp. 271-294.

[11] Z. Guo, Y. Fan, A Stochastic Multi-agent Optimization Model for Energy Infrastructure Planning under Uncertainty in An Oligopolistic Market, Networks and Spatial Economics 17 (2017), pp. 581-609.

[12] Y. Gürel, R. Güllü, Effect of a secondary market on a system with random demand and uncertain costs, International Journal of Production Economics, in press, https: //doi.org/10.1016/j.ijpe.2018.05.010.

[13] J. Gwinner, F. Raciti, On a class of Random Variational Inequalities on Random Sets, Numerical Functional Analysis and Optimization 27 (2006), pp. 619-636.

[14] J. Gwinner, F.Raciti, On monotone variational inequalities with random data, Journal of Mathematical Inequalities 3 (2009), pp. 443-453.

[15] J. Gwinner, F. Raciti, Some equilibrium problems under uncertainty and random variational inequalities, Annals of Operations research 200 (2012), pp. 299-319.

[16] B. Jadamba, A.A. Khan, F. Raciti, Regularization of Stochastic Variational Inequalities and a Comparison of an $L_{p}$ and a Sample-Path Approach, Nonlinear Analysis 94 (2014), pp. 65-83.

[17] B. Jadamba, F. Raciti, Variational Inequality Approach to Stochastic Nash Equilibrium Problems with an Application to Cournot Oligopoly, Journal of Optimization Theory and Applications 165 (2015), pp. 1050-1070.

[18] A. Jofré, R. J.-B. Wets, Variational convergence of bifunctions: motivating applications, SIAM Journal on Optimization 24 (2014), pp. 1952-1979.

[19] P.L. Kunsch, J. Springael , J.-P. Brans, The zero-emission certificates: A novel $\mathrm{CO}_{2-}$ pollution reduction instrument applied to the electricity market, European Journal of Operational Research 153 (2004), pp. 386-399.

[20] A. Maugeri, F. Raciti, On existence theorems for monotone and nonmonotone variational inequalities, Journal of Convex Analysis 16 (2009), pp. 899-911.

[21] A. Nagurney, Network Economics: A Variational Inequality Approach, Kluwer, Dordrecht, 1993.

[22] A. Nagurney, D. Matsypura, A supply chain network perspective for electric power generation, supply, transmission and consumption. In: Optimisation, econometric and financial analysis, E.J. Kontoghiorges and C. Gatu, (eds), Springer, Berlin, 2006, pp. $3-27$. 
[23] G. Oggioni, Y. Smeers, E. Allevi, S. Schaible, A Generalized Nash Equilibrium Model of Market Coupling in the European Power System, Networks and Spatial Economics 12 (2012), pp. 503-560.

[24] E. Pennings, Price or Quantity Setting under Uncertain Demand and Capacity Constraints: An Examination of the Profits, Journal of Economics 74 (2001), pp. 157-171.

[25] A. Prékopa, Stochastic Programming, Springer, Dordrecht, 1995.

[26] C. Ruiz, A.J. Conejo, J. D. Fuller, S.A. Gabriel, B. Hobbs, A tutorial review of complementarity models for decision-making in energy markets, EURO Journal on Decision Processes 2 (2014), pp. 91-120.

[27] A. Tolis, The effect of long-term expansion on the evolution of electricity price: numerical analysis of a theoretically optimised electricity market, European Journal of Operational Research 244 (2015), pp. 939-954. 